\title{
On a Class of Hierarchical Formations of Unicycles and Their Internal Dynamics
}

\author{
Luca Consolini, Member, IEEE, Fabio Morbidi, Associate Member, IEEE, Domenico Prattichizzo, Member, IEEE, \\ and Mario Tosques
}

\begin{abstract}
This paper studies a class of hierarchical formations for an ordered set of $n+1$ unicycle robots: the first robot plays the role of the leader and the formation is induced through a constraint function $F$, so that the position and orientation of the $i$ th robot depends only on the pose of the preceding ones. We study the dynamics of the formation with respect to the leader's reference frame by introducing the concept of reduced internal dynamics, we characterize its equilibria and provide sufficient conditions for their existence. The discovered theoretical results are applied to the case in which the constraint $F$ induces a formation where the $i$ th robot follows a convex combination of the positions of the previous $i-1$ vehicles. In this case, we prove that if the curvature of the leader's trajectory is sufficiently small, the positions and orientations of the robots, relative to the leader's reference frame, are confined in a precise polyhedral region.
\end{abstract}

Index Terms-Formation control, mobile robots, motion control, multiagent systems, nonlinear systems.

\section{INTRODUCTION}

$\mathbf{O}$ VER the past few years, multi-agent systems and cooperative control have been the subject of extensive research in the control and robotics communities [1]-[5]. The reasons for this success must be sought in multiple factors, such as, e.g., in the wider availability of wireless communication technologies and low-cost processing units, in the growing interest in parallel and embedded computing, in the definition of increasingly demanding tasks requiring the coordinate action of multiple autonomous agents to be successfully accomplished.

Due to its wide applicability in real-world scenarios (e.g., in patrolling/reconnaissance, entrapping/escorting, map building and exploration missions), the formation control problem has attracted considerable attention in the multi-agent systems literature (a list of representative papers, yet far from being complete, is [6]-[12]). The idea behind formation control is that of

Manuscript received November 05, 2009; revised April 16, 2010, November 19, 2010, April 14, 2011, and April 15, 2011; accepted July 07, 2011. Date of publication August 30, 2011; date of current version March 28, 2012. Recommended by Associate Editor J. Cortes.

L. Consolini is with the Department of Information Engineering, University of Parma, Parma 43100, Italy (e-mail: lucac@ce.unipr.it).

F. Morbidi is with the Institute for Design and Control of Mechatronical Systems, Johannes Kepler University, A-4040 Linz, Austria (e-mail: fabio. morbidi@jku.at).

D. Prattichizzo is with the Department of Information Engineering, University of Siena, 53100 Siena, Italy (e-mail: prattichizzo@ dii.unisi.it).

M. Tosques is with the Department of Civil Engineering, University of Parma, Parma 43100, Italy (e-mail: mario.tosques@unipr.it).

Color versions of one or more of the figures in this paper are available online at http://ieeexplore.ieee.org.

Digital Object Identifier 10.1109/TAC.2011.2166299

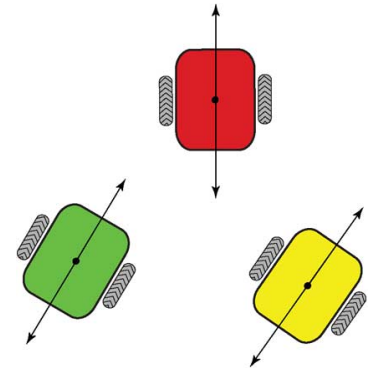

Fig. 1. If the initial configuration of a team of unicycles is the one shown in the figure, the vehicles cannot move as a single rigid body.

controlling the relative position and orientation of the agents in a group, while allowing the group to move as a whole [13].

Three major research lines in formation control have been developed in the last decade: behavior-based [14], [15], virtual structures [16]-[18], and leader-follower [13], [19]-[21].

Recently, the interest in formation control has been awakened by the introduction of the original notion of rigidity. Moving from the seminal papers [22], [23], Anderson and coworkers have started to systematically apply the rigid graph theory [24] to the analysis of formations of autonomous agents and they have shown the relevance of the rigidity concept in sensor network localization problems [25] as well as in other branches of engineering [26]-[28].

In [29] and [30], graph rigidity ideas have been used to design decentralized gradient control laws for the stabilization of a group of kinematic points to a target formation. Additionally, in [31], a distributed algorithm that stabilizes the shape of a relative sensing network to a desired formation has been proposed: the algorithm relies on the global minimization of the "stress majorization function" (a tool from multidimensional scaling theory) associated to the network.

Note that differently from formations of fully actuated agents as those considered in [29]-[31], given a team of unicycle robots, it is in general impossible to guarantee that the vehicles move as a single rigid body for an arbitrary initial condition, since the nonholonomic constraints must be satisfied. This problem is illustrated in Fig. 1, where the robots have different orientations and they cannot move as a rigid body since this would violate the nonholonomic constraints.

In this respect, the study of the relative dynamics of the robots, is of paramount importance to prevent undesired phenomena, such as uncontrolled oscillations or instabilities in the formation shape when the robots must obey some coordination rule during their motion.

Dealing with the problem of unit-speed unicycles coordination and assuming an all-to-all communication, the authors in 
[32] have shown an interesting connection between coupled oscillator theory (and notably Kuramoto model of coupled oscillators [33]) and control of multiple nonholonomic vehicles. The results in [32] have been extended in several directions in recent years. In [34], the case of limited communication topology is considered and in [35] the design methodology developed in [32] is adapted to the trajectory-tracking case. Finally, in [36] the authors have investigated the effect of few leader agents on the controllability of a heterogeneous group of unit-speed unicycles obeying a controlled sinusoidal phase-coupling protocol.

In this paper, we study a class of hierarchical formations for an ordered set of $n+1$ unicycle robots, where the first robot plays the role of the leader. The desired formation is obtained by imposing that the positions and orientations of the robots belong to a constraint which is the zero set of an assigned map $F$. Actually, the idea of defining a robot formation through the enforcement of a constraint is not new in the literature. In fact, there are similarities between the constraint function $F$ defined in this paper and the formation constraint function considered in [17], the concept of formation constraints in [7], and the abstraction and the goal function introduced in [37] and [12], respectively.

With respect to the above references, we make some extra assumptions on the constraint $F$ that allow the induced formation to possess some specific properties. In particular, to consider hierarchical formations, we assume that $F$ belongs to a family of maps in which the variables are the positions and the orientations of the robots, and the state of the $i$ th robot depends only on the states of the preceding ones. This may represent a unifying approach to study formations of unicycles with hierarchical structures and allow to find common properties in different control scenarios.

We present conditions on the map $F$ which guarantee that, for any trajectory followed by the leader, there exist unique controls for the followers (i.e., unique linear and angular velocities) such that they are asymptotically in formation (i.e., the constraint function is asymptotically satisfied). In this way, the behavior of the formation is enforced by prescribing a constraint function $F$ and the control law is entirely derived from it, and not assigned $a$ priori.

Moreover, the dynamics of the formation with respect to the leader's reference frame is studied by introducing the concept of reduced motion of the follower robots, which describes the motion of the followers in the leader's frame. The evolution of the reduced motion is governed by the reduced internal dynamics system (note that this concept is similar to the use of "shape variables" in [38]).

An equilibrium point of the reduced internal dynamics represents a configuration in which the reduced motion is constant, that is the robots' relative positions and orientations are constant. This is actually the only case in which the formation moves as a single rigid body. The main contribution of this paper consists in characterizing these equilibrium configurations and in providing sufficient conditions for their existence, that depend only on the constraint function $F$ and not on the specific choice of the control law.

In particular, a first theorem in Section II, states that if the vehicles are in formation, the relative position and the orientation of each follower with respect to the leader's reference frame are constant if and only if the leader and the followers either move along circular paths or parallel straight lines. Moreover, a second theorem shows that an equilibrium configuration always exists if the leader moves along a circle of sufficiently small curvature or along a straight line.

The results of these two theorems are applied in Section III to a specific constraint function $F$ that induces a formation where the $i$ th robot follows a convex combination of the positions of the preceding $i-1$ vehicles. This generalizes the hierarchical formations investigated in our previous works [39], [40]. For this specific case, a third theorem presents an invariance result for the internal dynamics of the formation, which provides sufficient conditions guaranteeing that, if the curvature of the leader's trajectory is sufficiently small, the positions and orientations of the follower robots, relative to the leader's reference frame, although not fixed, are confined in a precise polyhedral region.

Simulation results relative to the constraint function studied in Section III are presented in Section IV to illustrate the theory. Finally, in Section V, the main contributions of the paper are summarized and some concluding remarks are provided.

\section{A. Notation}

The following notation is used through the paper: $S^{1}$ denotes the quotient space $S^{1}=\mathbb{R} / 2 \pi \mathbb{Z}$, where $\mathbb{Z}$ is the set of the integer numbers; $\forall x=\left(x_{1}, x_{2}, \ldots, x_{n}\right)^{T}, y=\left(y_{1}, y_{2}, \ldots, y_{n}\right)^{T} \in$ $\mathbb{R}^{n}(n \geq 1),\langle x, y\rangle=\sum_{i=1}^{n} x_{i} y_{i},\|x\|=\sqrt{\langle x, x\rangle} ; \forall \theta \in S^{1}$, $\tau(\theta)=(\cos \theta, \sin \theta)^{T}, \eta(\theta)=(-\sin \theta, \cos \theta)^{T}$; if $\bar{x} \in \mathbb{R}^{n}$, $B(\bar{x}, \epsilon)=\left\{x \in \mathbb{R}^{n} \mid\|x-\bar{x}\| \leq \epsilon\right\} ;$ for any $(x, y)^{T} \in \mathbb{R}^{2} \backslash$ $\left\{(0,0)^{T}\right\}, \arg \left((x, y)^{T}\right)$ is the unique $\left.\left.\theta \in\right]-\pi, \pi\right]$ such that $(x, y)^{T}=\left\|(x, y)^{T}\right\| \tau(\theta)$.

Given a differentiable manifold $\mathcal{M}, T_{x} \mathcal{M}$ denotes the tangent space of $\mathcal{M}$ at $x \in \mathcal{M}$; given two functions $f(t)$ and $g(t)$, $(f \circ g)(t)=f(g(t))$ indicates their composition. If $\Omega$ is an open subset of $\mathbb{R}^{n}, \bar{x} \in \Omega$ and $F: \Omega \rightarrow \mathbb{R}^{m}$ is a differentiable map in $\bar{x}$, we denote by $F^{\prime}(\bar{x})$ the $m \times n$ Jacobian matrix of $F$ at $\bar{x}$. Moreover, we say that $F \in \mathcal{C}^{1,1}\left(\Omega, \mathbb{R}^{m}\right)$ if $F \in \mathcal{C}^{1}\left(\Omega, \mathbb{R}^{m}\right)$ and $F^{\prime}$ is a Lipschitz map.

\section{HierarchicAl Formations of Unicycle Robots}

Consider the following definition of robot as a velocity-controlled unicycle model [39].

Definition 1: A function $r=(x, y, \theta)^{T} \in \mathcal{C}^{1}\left(\left[0,+\infty\left[, \mathbb{R}^{2} \times\right.\right.\right.$ $S^{1}$ ) is called a unicycle robot (or simply a robot) if there exists a control function $(v, \omega)^{T} \in \mathcal{C}^{0}\left(\left[0,+\infty\left[, \mathbb{R}^{2}\right)\right.\right.$ such that

$$
\frac{d}{d t}\left(\begin{array}{l}
x \\
y \\
\theta
\end{array}\right)=\left(\begin{array}{cc}
\cos \theta & 0 \\
\sin \theta & 0 \\
0 & 1
\end{array}\right)\left(\begin{array}{l}
v \\
\omega
\end{array}\right) .
$$

For any $t \in\left[0,+\infty\left[,(x(t), y(t))^{T}\right.\right.$ represents the position of the robot at time $t, \theta(t)$ its heading, $\tau(\theta(t))$ the normalized velocity vector and $\eta(\theta(t))$ the normalized vector orthogonal to $\tau(\theta(t))$. Hence, $\{\tau(\theta(t)), \eta(\theta(t))\}$ represents the robot reference frame at time $t$ (see Fig. 2).

The next definition introduces an ordered set of $n+1$ robots, with $n \geq 1$. 


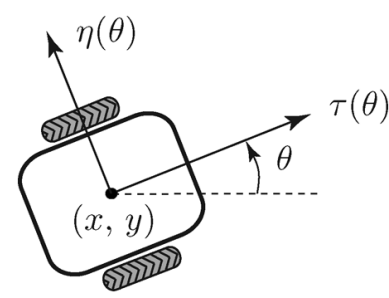

Fig. 2. Variables of the unicycle robot.

Definition 2: Let

$$
\begin{aligned}
\mathcal{X}= & \left(\mathbb{R}^{2} \times S^{1}\right)^{n+1} \\
= & \left\{\xi=\left(\xi_{0}, \xi_{1}, \ldots, \xi_{n}\right) \mid \xi_{i}=\left(x_{i}, y_{i}, \theta_{i}\right)^{T} \in \mathbb{R}^{2} \times S^{1},\right. \\
& \forall i=0, \ldots, n\} .
\end{aligned}
$$

A $\mathcal{C}^{1}$ map $\mathcal{R}:[0,+\infty[\longrightarrow \mathcal{X}$, defined by

$$
\mathcal{R}(t)=\left(r_{0}(t), r_{1}(t), \ldots, r_{n}(t)\right)
$$

where $r_{i}=\left(x_{i}, y_{i}, \theta_{i}\right)^{T}$ is a robot $\forall i=0,1, \ldots, n$, is called an $(n+1)$-tuple of robots. The set $\mathcal{X}$ is called the configuration space, $r_{0}$ the leader, and $r_{1}, r_{2}, \ldots, r_{n}$ the followers.

The following definition characterizes the class of constraints considered in this paper to define a formation of an $(n+1)$-tuple of robots.

Definition 3: Let $F$ be a map given by $F=$ $\left(F_{1}, F_{2}, \ldots, F_{n}\right)^{T}$, where $F_{i} \in \mathcal{C}^{1,1}\left(\mathcal{X}, \mathbb{R}^{2}\right), \forall i=1, \ldots, n$. The map $F$ is called a hierarchical constraint function if

$$
\begin{aligned}
\mathcal{F} & =\{\xi \in \mathcal{X} \mid F(\xi)=0\} \text { is not empty and } \\
\partial_{\xi_{j}} F_{i}(\xi) & =0, \forall \xi \in \mathcal{X}, i=1, \ldots, n-1, j=i+1, \ldots, n
\end{aligned}
$$

that is, every $F_{i}$ depends only on $\left(\xi_{0}, \ldots, \xi_{i}\right)$. For the sake of brevity, we henceforth refer to $F$ simply as constraint function. The set $\mathcal{F}$ is called the constraint set: it is the set of the configurations $\xi$ compatible with the constraint function $F$.

The following definition introduces the notion of formation of an $(n+1)$-tuple of robots used throughout the paper.

Definition 4: Let $F$ be a constraint function. An $(n+1)$-tuple of robots $\mathcal{R}$ is said in $F$-formation if

$$
F(\mathcal{R}(t))=0, \quad \forall t \geq 0
$$

that is, if $\mathcal{R}(t) \in \mathcal{F}, \forall t \geq 0$.

As illustrated in the example of Fig. 3, if an $(n+1)$-tuple of robots is in $F$-formation, the motion of each follower depends only on the behavior of the preceding robots. This is a consequence of the triangular structure of the function $F$, where every component $F_{i}$ depends only on the position and the orientation of the robots that have index less or equal than $i$. In this way, $F$-formations represent a generalization of standard leader-follower architectures, where the motion of each robot depends only on the behavior of one of the preceding vehicles (see for instance [40]).

To provide concise statements in the following, let us introduce the vectors $v_{F}=\left(v_{1}, v_{2}, \ldots, v_{n}\right)^{T}, \omega_{F}=$ $\left(\omega_{1}, \omega_{2}, \ldots, \omega_{n}\right)^{T}$, where $v_{i}, \omega_{i}$ are the control inputs of

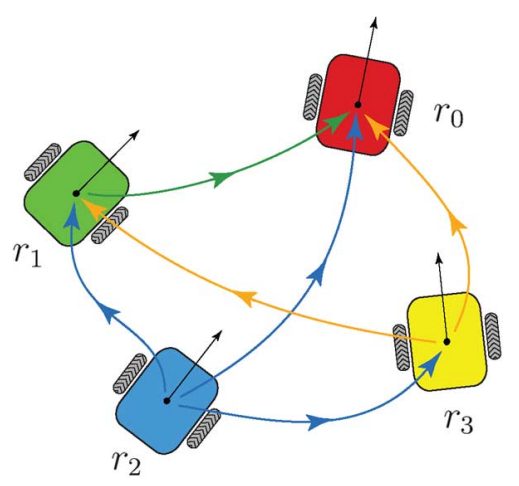

Fig. 3. In an $F$-formation, the robots are ordered: the first robot $r_{0}$ is the leader and each of the followers $r_{1}, r_{2}, r_{3}$ bases its motion on the behavior of the preceding robots, as shown by the colored arrows.

the $i$ th robot. In this way the kinematic equations of the $n+1$ robots can be written in the form

$$
\dot{\xi}=g\left(\xi, v_{0}, \omega_{0}, v_{F}, \omega_{F}\right)
$$

where $g=\left(g_{0}, g_{1}, \ldots, g_{n}\right)^{T}$ is such that $\forall i=0, \ldots, n$ :

$$
g_{i}\left(\xi, v_{0}, \omega_{0}, v_{F}, \omega_{F}\right)=\left(\begin{array}{cc}
\cos \theta_{i} & 0 \\
\sin \theta_{i} & 0 \\
0 & 1
\end{array}\right)\left(\begin{array}{c}
v_{i} \\
\omega_{i}
\end{array}\right) .
$$

The following proposition states that $\mathcal{F}$ is a manifold and is controlled invariant for the closed-loop system (5) under the key assumption (6) (see below). Condition (6) requires that the directional derivative of $F_{i}$ along the two vector fields $\left(\cos \theta_{i}, \sin \theta_{i}, 0\right)^{T}$ and $(0,0,1)^{T}$ is full rank, $\forall i=1, \ldots, n$. As it will be shown in the proof, this guarantees that it is always possible to find feedback controls $v_{F}, \omega_{F}$ such that $\mathcal{F}$ is an invariant manifold for the closed-loop system (5).

Proposition 1: Let $F$ be a constraint function and suppose that $\forall \xi \in \mathcal{F}, \forall i=1, \ldots, n$

$$
\operatorname{det}\left(\cos \theta_{i} \partial_{x_{i}} F_{i}(\xi)+\sin \theta_{i} \partial_{y_{i}} F_{i}(\xi), \partial_{\theta_{i}} F_{i}(\xi)\right) \neq 0 .
$$

Then the following facts hold:

1) $\mathcal{F}$ is a differentiable manifold of dimension $n+3$.

2) For each $\xi \in \mathcal{F}$ and for each $\left(v_{0}, \omega_{0}\right)^{T} \in \mathbb{R}^{2}$, there exist unique controls $v_{F}\left(\xi, v_{0}, \omega_{0}\right), \omega_{F}\left(\xi, v_{0}, \omega_{0}\right)$ such that $g\left(\xi, v_{0}, \omega_{0}, v_{F}\left(\xi, v_{0}, \omega_{0}\right), \omega_{F}\left(\xi, v_{0}, \omega_{0}\right)\right) \in T_{\xi} \mathcal{F}$.

3) For any $\left(v_{0}, \omega_{0}\right)^{T} \in \mathcal{C}^{0}\left(\left[0,+\infty\left[, \mathbb{R}^{2}\right), \forall \bar{\xi} \in \mathcal{F}\right.\right.$, the following property holds: $\mathcal{R}$ is an $(n+1)$-tuple of robots in $F$-formation such that $\mathcal{R}(0)=\bar{\xi}$ if and only if $\mathcal{R}$ is the unique solution of the following system:

$$
\left\{\begin{array}{l}
\dot{\xi}=g\left(\xi, v_{0}, \omega_{0}, v_{F}\left(\xi, v_{0}, \omega_{0}\right), \omega_{F}\left(\xi, v_{0}, \omega_{0}\right)\right) \\
\xi(0)=\bar{\xi}
\end{array}\right.
$$

In particular, if the $(n+1)$-tuple of robots is in $F$-formation at the initial time, then for any trajectory $\left(x_{0}, y_{0}, \theta_{0}\right)^{T}$ of the leader $r_{0}$, there exists a unique choice of controls $\left(v_{i}, \omega_{i}\right)^{T} \in$ $\mathcal{C}\left(\left[0,+\infty\left[, \mathbb{R}^{2}\right), i=1, \ldots, n\right.\right.$, such that $r_{1}, \ldots, r_{n}$ follow $r_{0}$ in $F$-formation.

Proof: Let $F^{\prime}$ be the Jacobian matrix of $F$. Since rank $F^{\prime}(\xi)=2 n$ by (6), we have that 1 ) holds by the implicit function theorem. To prove 2), set, $\forall i=1, \ldots, n$, 
$\forall j=0, \ldots, n, \partial_{\xi_{j}} F_{i}(\xi)=\left(\partial_{x_{j}} F_{i}(\xi), \partial_{y_{j}} F_{i}(\xi), \partial_{\theta_{j}} F_{i}(\xi)\right)$, which is a $2 \times 3$ matrix and $\forall i=1, \ldots, n$

$$
\begin{aligned}
A_{i j}(\xi) & =\partial_{\xi_{j}} F_{i}(\xi)\left(\begin{array}{cc}
\cos \theta_{j} & 0 \\
\sin \theta_{j} & 0 \\
0 & 1
\end{array}\right) \\
& =\left(\cos \theta_{j} \partial_{x_{j}} F_{i}(\xi)+\sin \theta_{j} \partial_{y_{j}} F_{i}(\xi), \partial_{\theta_{j}} F_{i}(\xi)\right) .
\end{aligned}
$$

Then, $\forall\left(v_{0}, \omega_{0}\right)^{T} \in \mathbb{R}^{2}$, let $v_{F}\left(\xi, v_{0}, \omega_{0}\right), \omega_{F}\left(\xi, v_{0}, \omega_{0}\right)$ be given by the unique solution of the following triangular system:

$$
A_{i i}(\xi)\left(\begin{array}{c}
v_{i} \\
\omega_{i}
\end{array}\right)=-\sum_{j=0}^{i-1} A_{i j}(\xi)\left(\begin{array}{c}
v_{j} \\
\omega_{j}
\end{array}\right), \quad i=1, \ldots, n
$$

which is solvable since $\operatorname{det} A_{i i}(\xi) \neq 0$ by (8) and hypothesis (6). Therefore 2) holds. Finally, 3) is a consequence of 2) and the existence and uniqueness of the solution of (7).

Remark 1: From (9) it follows that the control functions $v_{F}\left(\xi, v_{0}, \omega_{0}\right)=\left(v_{1}, v_{2}, \ldots, v_{n}\right)^{T}, \omega_{F}\left(\xi, v_{0}, \omega_{0}\right)=$ $\left(\omega_{1}, \omega_{2}, \ldots, \omega_{n}\right)^{T}$, appearing in 2$)$ of proposition 1 , can be computed iteratively as follows:

$$
\begin{aligned}
\left(\begin{array}{c}
v_{1} \\
\omega_{1}
\end{array}\right)= & -A_{11}^{-1}(\xi) A_{10}(\xi)\left(\begin{array}{c}
v_{0} \\
\omega_{0}
\end{array}\right) \\
\left(\begin{array}{c}
v_{2} \\
\omega_{2}
\end{array}\right)= & -A_{22}^{-1}(\xi)\left(A_{21}(\xi)\left(\begin{array}{l}
v_{1} \\
\omega_{1}
\end{array}\right)+A_{20}(\xi)\left(\begin{array}{l}
v_{0} \\
\omega_{0}
\end{array}\right)\right) \\
& \vdots \\
\left(\begin{array}{c}
v_{n} \\
\omega_{n}
\end{array}\right)= & -A_{n n}^{-1}(\xi)\left(\sum_{j=0}^{n-1} A_{i j}(\xi)\left(\begin{array}{c}
v_{j} \\
\omega_{j}
\end{array}\right)\right) .
\end{aligned}
$$

Note that the controls defined by (10) are not decentralized since $v_{i}, \omega_{i}$ depend on the states of all preceding robots $\xi_{j}, j=$ $1, \ldots, i$.

The following proposition deals with the stabilizability of the controlled invariant manifold $\mathcal{F}$. In particular, it shows that the regularity condition (11), which is slightly stronger than (6) (see also proposition 6 below), guarantees the local stabilizability of a robot formation. Roughly speaking, this means that if the robots are not initially "too far" from being in formation, we can always determine a control law for the followers that allows them to asymptotically achieve the desired formation, regardless of the trajectory of the leader.

Proposition 2: Suppose that $F$ is a constraint function such that there exists $\epsilon>0$, so that $\forall \xi \in \mathcal{F}(\epsilon), \forall i=1, \ldots, n$ :

$$
\operatorname{det}\left(\cos \theta_{i} \partial_{x_{i}} F_{i}(\xi)+\sin \theta_{i} \partial_{y_{i}} F_{i}(\xi), \partial_{\theta_{i}} F_{i}(\xi)\right) \neq 0
$$

where $\mathcal{F}(\epsilon)=\{\xi \in \mathcal{X} \mid\|F(\xi)\| \leq \epsilon\}$.

Then $\forall v_{0}, \omega_{0} \in \mathcal{C}([0,+\infty[, \mathbb{R})$, there exist control functions $v_{F}\left(\xi, v_{0}, \omega_{0}\right), \omega_{F}\left(\xi, v_{0}, \omega_{0}\right) \in \mathcal{C}\left(\left[0,+\infty\left[\times \mathcal{X}, \mathbb{R}^{n}\right)\right.\right.$ such that, $\forall \bar{\xi} \in \mathcal{F}(\epsilon)$, the solution $\mathcal{R}(t)$ of the initial value problem (7) is defined on $\left[0,+\infty\left[\right.\right.$ and is such that $\lim _{t \rightarrow+\infty}\|F(\mathcal{R}(t))\|=0$.
Proof: By hypothesis (11), using the notations of the proof of proposition 1, it suffices to define $v_{F}, \omega_{F}$ as the solution of the following system: $\forall i=1, \ldots, n$

$$
A_{i i}(\xi)\left(\begin{array}{c}
v_{i} \\
\omega_{i}
\end{array}\right)=-\sum_{j=0}^{i-1} A_{i j}(\xi)\left(\begin{array}{c}
v_{j} \\
\omega_{j}
\end{array}\right)-k F_{i}(\xi)
$$

with $k>0$. Since $\operatorname{det} A_{i i}(\xi) \neq 0$, by (8) and hypothesis (6) the control functions are given by $\forall i=1, \ldots, n$

$$
\left(\begin{array}{c}
v_{i} \\
\omega_{i}
\end{array}\right)=A_{i i}^{-1}(\xi)\left(-\sum_{j=0}^{i-1} A_{i j}(\xi)\left(\begin{array}{c}
v_{j} \\
\omega_{j}
\end{array}\right)-k F_{i}(\xi)\right) .
$$

Since $F$ is $\mathcal{C}^{1,1}$ by definition 3, the right-hand side of the closed-loop system (7) is continuous in the couple $(t, \xi)$ and globally Lipschitz as a function of $\xi$. Therefore, $\forall \bar{\xi} \in \mathcal{F}(\epsilon)$, $\forall t \geq 0, \forall i=1, \ldots, n$, the solution $\mathcal{R}(t)$ of system (7) is defined on $[0,+\infty[$ and, by (12), has the property

$$
\frac{d}{d t}\left(F_{i}(\mathcal{R}(t))\right)=-k F_{i}(\mathcal{R}(t)), \quad \forall t \geq 0, \forall i=1, \ldots, n
$$

which implies the thesis.

Remark 2: As in remark 1, the control functions $v_{F}\left(\xi, \omega_{0}, v_{0}\right)=\left(v_{1}, v_{2}, \ldots, v_{n}\right)^{T}, \omega_{F}\left(\xi, \omega_{0}, v_{0}\right)=$ $\left(\omega_{1}, \omega_{2}, \ldots, \omega_{n}\right)^{T}$ appearing in (13) can be computed iteratively in the following way:

$$
\begin{aligned}
& \left(\begin{array}{l}
v_{1} \\
\omega_{1}
\end{array}\right)=-A_{11}^{-1}(\xi)\left(A_{10}(\xi)\left(\begin{array}{c}
v_{0} \\
\omega_{0}
\end{array}\right)-k F_{1}\left(\xi_{0}, \xi_{1}\right)\right) \\
& \left(\begin{array}{c}
v_{2} \\
\omega_{2}
\end{array}\right)=-A_{22}^{-1}(\xi)\left(A_{21}(\xi)\left(\begin{array}{l}
v_{1} \\
\omega_{1}
\end{array}\right)+A_{20}(\xi)\left(\begin{array}{l}
v_{0} \\
\omega_{0}
\end{array}\right)\right. \\
& \text { - } \left.k F_{2}\left(\xi_{0}, \xi_{1}, \xi_{2}\right)\right) \\
& \left(\begin{array}{c}
v_{n} \\
\omega_{n}
\end{array}\right)=-A_{n n}^{-1}(\xi)\left(\sum_{j=0}^{n-1} A_{i j}(\xi)\left(\begin{array}{c}
v_{j} \\
\omega_{j}
\end{array}\right)-k F_{n}(\xi)\right) .
\end{aligned}
$$

In (12), the two terms appearing on the right-hand side have the following meaning: the term $-\sum_{j=0}^{i-1} A_{i j}(\xi)\left(\begin{array}{l}v_{j} \\ \omega_{j}\end{array}\right)$ is the one which makes the constraint set $\mathcal{F}$ a controlled invariant, while $\left.-k F_{i}(\xi)\right)$ is the stabilizing term, which is null on the constraint set $\mathcal{F}$.

Remark 3: Note that in order to achieve finite-time convergence to the constraint set $\mathcal{F}$ starting from any $\bar{\xi} \in \mathcal{F}(\epsilon)$, it is sufficient to consider the solution of system (7) with the controls defined by $\forall i=1, \ldots, n$

$$
\left(\begin{array}{c}
v_{i} \\
\omega_{i}
\end{array}\right)=A_{i i}^{-1}(\xi)\left(-\sum_{j=0}^{i-1} A_{i j}(\xi)\left(\begin{array}{c}
v_{j} \\
\omega_{j}
\end{array}\right)-k \sigma\left(F_{i}(\xi)\right)\right)
$$

where, for any vector $w=\left(w_{1}, w_{2}\right)^{T} \in \mathbb{R}^{2}$ we set

$$
\sigma(w)=\left(\sqrt{\left|w_{1}\right|} \operatorname{sign} w_{1}, \sqrt{\left|w_{2}\right|} \operatorname{sign} w_{2}\right)^{T} .
$$

Then, $\forall i=1, \ldots, n$ we have that

$$
\frac{d}{d t}\left(F_{i}(\mathcal{R}(t))\right)=-k \sigma\left(F_{i}(\mathcal{R}(t))\right), \quad \forall t \geq 0, \forall i=1, \ldots, n
$$


which implies that $\forall i=1, \ldots, n, h=1,2$

$$
\left|F_{i h}(\mathcal{R}(t))\right|= \begin{cases}\frac{\left(2 \sqrt{\left|F_{i h}(\bar{\xi})\right|}-k t\right)^{2}}{4}, & \text { if } t \leq \frac{2 \sqrt{\left|F_{i h}(\bar{\xi})\right|}}{k} \\ 0, & \text { if } t>\frac{2 \sqrt{\left|F_{i h}(\bar{\xi})\right|}}{k}\end{cases}
$$

being $\forall i=1, \ldots, n, F_{i}(\xi)=\left(F_{i 1}(\xi), F_{i 2}(\xi)\right)^{T}$. Therefore the solution reaches the constraint set within the time

$$
\bar{t}=\max \left\{\frac{2 \sqrt{F_{i h}(\bar{\xi})}}{k}, i=1, \ldots, n, h=1,2\right\}
$$

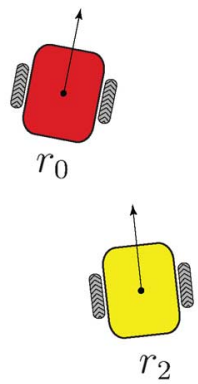

$r_{2}$

2

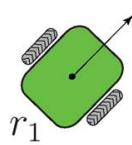

Fig. 4. If the constraint function $F$ is rototranslation invariant, the robots in (a) are in $F$-formation if and only if the robots in (b) are in $F$-formation, since the team in (a) can be superimposed to the team in (b) via a rototranslation.

Note that $G$ is a rototranslation invariant function if and only if for any $\xi \in \mathcal{X}, G$ is constant on the set $\llbracket \xi \rrbracket$.

In the case of rototranslation invariant constraint functions, it is natural to define a reduced constraint set as follows.

Definition 8: The reduced constraint set $\mathcal{F}_{r}$ is the set $\mathcal{F}_{r}=$ $\mathcal{F} / \sim$.

In this way, each element of $\mathcal{F}_{r}$ represents a set of configurations for the formation that differ only by a rototranslation.

Given an $(n+1)$-tuple of robots $\mathcal{R}$, the reduced motion of $\mathcal{R}$ is the map $\llbracket \mathcal{R} \rrbracket:[0,+\infty[\longrightarrow \mathcal{X} / \sim$ defined by $\llbracket \mathcal{R} \rrbracket(t)=$ $\llbracket \mathcal{R}(t) \rrbracket, \forall t \geq 0$. Note that $\llbracket \mathcal{R} \rrbracket$ describes the motion of the followers in the leader's reference frame.

The following proposition presents some properties of the reduced motion of an $(n+1)$-tuple of robots.

Proposition 3: Let $F$ be a rototranslation invariant function.

1) Let $\mathcal{R}=\left(r_{0}, \ldots, r_{n}\right)$ be an $(n+1)$-tuple of robots. The reduced motion $\llbracket \mathcal{R} \rrbracket$ of $\mathcal{R}$ is constant if and only if there exists $\bar{\xi} \in \mathcal{F}$ such that

$$
H_{-r_{0}(t)}(\mathcal{R}(t))=\bar{\xi}, \quad \forall t \geq 0 .
$$

This means that the position and the orientation of the followers, with respect to the leader's reference frame, is fixed.

2) Let $\mathcal{R}_{1}, \mathcal{R}_{2}$ be two $(n+1)$-tuples of robots in $F$-formation, with the same controls $v_{0}$ and $\omega_{0}$ for the leader. Suppose that

$$
\mathcal{R}_{1}(0) \sim \mathcal{R}_{2}(0)
$$

then

$$
\mathcal{R}_{1}(t) \sim \mathcal{R}_{2}(t), \quad \forall t \geq 0
$$

that is, if $\llbracket \mathcal{R}_{1}(0) \rrbracket=\llbracket \mathcal{R}_{2}(0) \rrbracket$, then

$$
\llbracket \mathcal{R}_{1}(t) \rrbracket=\llbracket \mathcal{R}_{2}(t) \rrbracket, \quad \forall t \geq 0 .
$$

Proof: Part 1) of the statement is evident. For part 2), by (18) there exists $p \in \mathbb{R}^{2} \times S^{1}$, such that

Proving (19) is equivalent to show that

$$
H_{p}\left(\mathcal{R}_{1}(t)\right)=\mathcal{R}_{2}(t), \quad \forall t \geq 0 .
$$

$$
H_{p}\left(\mathcal{R}_{1}(0)\right)=\mathcal{R}_{2}(0) .
$$


In fact $F\left(H_{p}\left(\mathcal{R}_{1}(t)\right)\right)=0$ since $F$ is rototranslation invariant, moreover $H_{p}\left(\mathcal{R}_{1}(t)\right)$ is still an $(n+1)$-tuple of robots. Then (20) holds by 3 ) of proposition 1 .

Remark 6: Let $F$ be a rototranslation invariant constraint function. Set

$$
\Gamma_{F}=\left\{\xi=\left(\xi_{0}, \ldots, \xi_{n}\right)^{T} \in \mathcal{X} \mid \xi_{0}=0, F(\xi)=0\right\} .
$$

Then the map $\mathcal{J}: \mathcal{F}_{r} \longrightarrow \Gamma_{F}$ defined by

$$
\mathcal{J}(\llbracket \xi \rrbracket)=H_{-\xi_{0}}(\xi)
$$

is a bijection. Moreover an $(n+1)$-tuple of robots $\mathcal{R}$ is in $F$-formation if and only if $H_{-r_{0}(t)}(\mathcal{R}(t)) \in \Gamma_{F}, \forall t \geq 0$.

As suggested by (22), $\mathcal{F}_{r}$ is the set of all configurations of the followers in the leader's reference frame, that are compatible with the constraint function.

The following proposition states that if $F$ is a rototranslation invariant constraint function, then each follower can compute its control input by only knowing the pose of the preceding robots in its own reference frame. In this way, the control law of the followers can be implemented by relying on the information provided by on-board sensors.

Proposition 4: If $F$ is rototranslation invariant, then for any $i=1, \ldots, n, j=0, \ldots, n$ and for any $p \in \mathbb{R}^{2} \times S^{1}$

$$
A_{i j}(\xi)=A_{i j}\left(H_{p}(\xi)\right), \quad \forall \xi \in \mathcal{X}
$$

i.e., terms $A_{i j}$ are constant on the equivalence classes of $X / \sim$. Therefore the controls $v_{i}, \omega_{i}$ given by (10), (13), and (15) do not change by replacing $\xi$ with $H_{-\xi_{i}}(\xi)$, i.e., they can be expressed just in terms of the coordinates relative to the reference frame of the $i$ th robot.

Proof: Set $p=(\bar{x}, \bar{y}, \bar{\theta})^{T} \in \mathbb{R}^{2} \times S^{1}, \forall j=0, \ldots, n$. Since $F$ is rototranslation invariant, $\forall i=1, \ldots, n, \forall \eta \in\left(\mathbb{R}^{2} \times S^{1}\right)^{n}$

$$
\begin{aligned}
F_{i}^{\prime}(\xi) \eta & =\lim _{h \rightarrow 0} \frac{F_{i}(\xi+h \eta)-F_{i}(\xi)}{h} \\
& =\lim _{h \rightarrow 0} \frac{F_{i}\left(H_{p}(\xi+h \eta)\right)-F_{i}\left(H_{p}(\xi)\right)}{h} \\
& =\lim _{h \rightarrow 0} \frac{F_{i}\left(H_{p}(\xi)+h H_{p}^{\prime}(\xi) \eta\right)-F_{i}\left(H_{p}(\xi)\right)}{h} \\
& =F_{i}^{\prime}\left(H_{p}(\xi)\right) H_{p}^{\prime}(\xi) \eta
\end{aligned}
$$

where $H_{p}^{\prime}(\xi)$ is the block-diagonal matrix with $(n+1)$ blocks given by the constant $3 \times 3$ matrix $\left(\begin{array}{cc}R(\bar{\theta}) & (0,0)^{T} \\ (0,0) & 1\end{array}\right)$. For any $j=0, \ldots, n$, set

$$
\begin{aligned}
\eta_{j} & =\left(0, \ldots,(0,0,1)_{j}, 0, \ldots, 0\right)^{T} \in\left(\mathbb{R}^{2} \times S^{1}\right)^{n+1}, \\
\zeta_{j} & =\left(0, \ldots,(\cos \bar{\theta}, \sin \bar{\theta}, 0)_{j}, 0, \ldots, 0\right)^{T} \in\left(\mathbb{R}^{2} \times S^{1}\right)^{n+1}
\end{aligned}
$$

then by (24)

$$
\begin{aligned}
A_{i j}(\xi)= & \left(F_{i}^{\prime}(\xi) \zeta_{j}, F_{i}^{\prime}(\xi) \eta_{j}\right) \\
= & \left(F_{i}^{\prime}\left(H_{p}(\xi)\right) H_{p}^{\prime}(\xi) \zeta_{j}, F_{i}^{\prime}\left(H_{p}(\xi)\right) H_{p}^{\prime}(\xi) \eta_{j}\right) \\
= & \left(\cos \left(\theta_{j}+\bar{\theta}\right) \partial_{x_{j}} F_{i}\left(H_{p}(\xi)\right)\right. \\
& \left.\quad+\sin \left(\theta_{j}+\bar{\theta}\right) \partial_{y_{j}} F_{i}\left(H_{p}(\xi)\right), \partial_{\theta_{j}} F_{i}\left(H_{p}(\xi)\right)\right) \\
= & A_{i j}\left(H_{p}(\xi)\right) .
\end{aligned}
$$

Since $\mathcal{F}_{r}$ is the quotient set of $\mathcal{F}$ by the equivalence relation $\sim$, it has a lower dimension than $\mathcal{F}$ as specified in the following proposition.

Proposition 5: If $F$ is a regular rototranslation invariant constraint function, $\mathcal{F}_{r}$ is a differentiable manifold of dimension $n$, diffeomorphic to $\Gamma_{F}$.

Proof: By (6) and the implicit function theorem, the subset of $\mathcal{F}$ given by $\Gamma_{F}$ [see (21)] is a submanifold of dimension $n$ of $\mathcal{X}$. Since the map (22) is a bijection, this induces a natural structure of differentiable manifold on $\mathcal{F}_{r}$.

In the following definition, $\rho_{F}$ represents the minimum radius of a circle centered at the leader, which contains all the positions that the followers can assume while respecting the constraint $F$.

Definition 9: For any $i=1, \ldots, n$, let $\Pi_{i}: \mathcal{X} \rightarrow \mathbb{R}^{2}$ be the map defined by $\Pi_{i}(\xi)=\left(x_{i}, y_{i}\right)^{T}$ and let $F$ be a rototranslation invariant constraint function. Set

$$
\rho_{F}=\sup \left\{\left\|\Pi_{i}(\xi)\right\| \mid \xi \in \Gamma_{F}\right\}
$$

which is the radius of the smallest circle centered at $(0,0)^{T}$ and containing all $\left(x_{i}, y_{i}\right)^{T}=\Pi_{i}(\xi)$, for any $i=1, \ldots, n$, and all possible configuration $\xi \in \Gamma_{F}$, that is

$$
\bigcup_{i=1}^{n} \Pi_{i}\left(\Gamma_{F}\right) \subset B\left((0,0)^{T}, \rho_{F}\right) \text {. }
$$

Proposition 6: Let $F$ be regular and rototranslation invariant. If $\rho_{F}<+\infty$, then there exists $\epsilon>0$ such that, $\forall \xi \in \mathcal{F}(\epsilon)$, $\forall i=1, \ldots, n$

$$
\operatorname{det}\left(\cos \theta_{i} \partial_{x_{i}} F_{i}(\xi)+\sin \theta_{i} \partial_{y_{i}} F_{i}(\xi), \partial_{\theta_{i}} F_{i}(\xi)\right) \neq 0
$$

i.e., hypothesis (11) of proposition 2 is satisfied.

Proof: Set $\Gamma_{F}$ is compact since it is a closed subset of $\left(B\left((0,0)^{T}, \rho_{F}\right) \times S^{1}\right)^{n}$ by the definition of $\rho_{F}$. Let $\Phi: \mathcal{X} \rightarrow$ $\left(\mathbb{R}^{2} \times S^{1}\right) \times \mathbb{R}^{2 n}$ be the map defined by $\Phi(\xi)=\left(\xi_{0}, F(\xi)\right)$, $\forall \xi \in \mathcal{X}$. Since $\Gamma_{F}=\{\xi \in \mathcal{X} \mid \Phi(\xi)=0\}$, condition (6) holds $\forall \xi \in \Gamma_{F}$ and $\Gamma_{F}$ is compact, then there exists an $\epsilon>0$ such that, $\forall \xi \in \Phi^{-1}(B(0, \epsilon))$

$$
\operatorname{det}\left(\cos \theta_{i} \partial_{x_{i}} F_{i}(\xi)+\sin \theta_{i} \partial_{y_{i}} F_{i}(\xi), \partial_{\theta_{i}} F_{i}(\xi)\right) \neq 0
$$

where $\Phi^{-1}(B(0, \epsilon))$ is the counter image of $B(0, \epsilon)=$ $\left\{\eta \in \mathbb{R}^{2 n+3} \mid\|\eta\| \leq \epsilon\right\}$. Since $\mathcal{F}(\epsilon)=\{\xi \in \mathcal{X}\|\mid F(\xi)\| \leq$ $\epsilon\}, \Phi^{-1}(B(0, \epsilon))=\left\{H_{-\xi_{0}}(\xi) \mid \xi \in \mathcal{F}(\epsilon)\right\}$, that is $\Phi^{-1}(B(0, \epsilon))$ is a set of representative elements of the equivalence classes $\llbracket \xi \rrbracket$ with $\xi \in \mathcal{F}(\epsilon)$. Moreover, by (23), $\operatorname{det}\left(\cos \theta_{i} \partial_{x_{i}} F_{i}(\xi)+\sin \theta_{i} \partial_{y_{i}} F_{i}(\xi), \partial_{\theta_{i}} F_{i}(\xi)\right)$ is constant on the elements $\xi$ of the same equivalence class, since $F$ is rototranslation invariant, therefore, $\forall \xi \in \mathcal{F}(\epsilon)$

$$
\operatorname{det}\left(\cos \theta_{i} \partial_{x_{i}} F_{i}(\xi)+\sin \theta_{i} \partial_{y_{i}} F_{i}(\xi), \partial_{\theta_{i}} F_{i}(\xi)\right) \neq 0
$$

by (26).

Let $F$ be a regular rototranslation invariant constraint function and let $\omega_{0} \in \mathcal{C}([0,+\infty[, \mathbb{R})$ be the control for the leader. A consequence of part 2) of proposition 3 is that the following map is well defined:

$$
\begin{aligned}
\Phi_{\omega_{0}}:\left[0,+\infty\left[\times \mathcal{F}_{r}\right.\right. & \longrightarrow \mathcal{F}_{r} \\
(t, \llbracket \zeta \rrbracket) & \rightsquigarrow \Phi_{\omega_{0}}(t, \llbracket \zeta \rrbracket)
\end{aligned}
$$




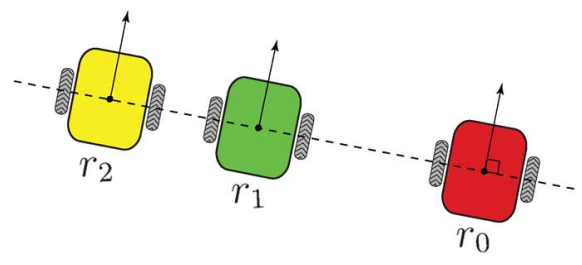

Fig. 5. For a three-robot formation, condition (30) prevents the robots $r_{0}, r_{1}, r_{2}$ from being all with the same orientation and aligned on a straight line orthogonal to their heading direction.

where

$$
\Phi_{\omega_{0}}(t, \llbracket \zeta \rrbracket)=\llbracket \xi(t) \rrbracket
$$

being $\xi$ the unique solution of the following system:

$$
\left\{\begin{array}{l}
\dot{\xi}=g\left(\xi, 1, \omega_{0}, v_{F}\left(\xi, 1, \omega_{0}\right), \omega_{F}\left(\xi, 1, \omega_{0}\right)\right) \\
\xi(0)=\zeta
\end{array}\right.
$$

where $g$ is given by (5) and $v_{F}, \omega_{F}$ by proposition 1 .

Definition 10: Set $\forall \omega_{0} \in \mathbb{R}, \forall \llbracket \xi \rrbracket \in \mathcal{F}_{r}$

$$
\Psi\left(\llbracket \xi \rrbracket, \omega_{0}\right)=\left.\frac{d}{d t}\left(\Phi_{\omega_{0}}(t, \llbracket \xi \rrbracket)\right)\right|_{t=0} \in T_{\mathcal{F}_{r}}(\llbracket \xi \rrbracket)
$$

where $T_{\mathcal{F}_{r}}(\llbracket \xi \rrbracket)$ is the tangent space of $\mathcal{F}_{r}$ at $\llbracket \xi \rrbracket$. Then $\forall \omega_{0} \in$ $\mathcal{C}\left(\left[0,+\infty[, \mathbb{R}), \forall \llbracket \zeta \rrbracket \in \mathcal{F}_{r}, \Phi_{\omega_{0}}(t, \llbracket \zeta \rrbracket)\right.\right.$ is the solution of the following system defined on the manifold $\mathcal{F}_{r}$ :

$$
\left\{\begin{array}{l}
\dot{\Phi}(t)=\Psi\left(\Phi(t), \omega_{0}(t)\right) \\
\Phi(0)=\llbracket \zeta \rrbracket
\end{array}\right.
$$

which we call the reduced internal dynamics of the formation. Thanks to the diffeomorphism between $\mathcal{F}_{r}$ and $\Gamma_{F}$, it represents the motion of the followers in the leader's reference frame.

As a consequence of (28), given $\llbracket \xi \rrbracket \in \mathcal{F}_{r}$ and $\omega_{0} \in \mathbb{R}$, $\left(\llbracket \xi \rrbracket, \omega_{0}\right)$ is an equilibrium point of $\Psi$, that is $\Psi\left(\llbracket \xi \rrbracket, \omega_{0}\right)=0$ if and only if the reduced motion $\llbracket \mathcal{R} \rrbracket$ of the $(n+1)$-tuple of robots $\mathcal{R}$ (which is in $F$-formation and $\mathcal{R}(0)=\xi$ ) is constant. Roughly speaking, the equilibria of $\Psi$ are the configurations in which the position and orientation of the followers are constant in the leader's reference frame.

The following theorem characterizes the $(n+1)$-tuple of robots $\mathcal{R}$ whose reduced motion $\llbracket R \rrbracket$ is constant. In particular, it shows that if condition (30) is satisfied, then the position and the orientation of each follower with respect to the leader's reference frame are fixed (i.e., the followers are stationary in the leader's frame) if and only if the leader and the followers either move along circular paths or parallel straight lines.

The nonalignment condition (30) means that the manifold $\mathcal{F}$ must not contain configurations in which all the followers are placed on a straight line passing through the leader and orthogonal to its heading direction $\tau\left(\theta_{0}\right)$, with the followers oriented as the leader (see Fig. 5).

Theorem 1: Suppose that $F$ is a regular rototranslation invariant constraint function and that $\mathcal{R}$ is an $(n+1)$-tuple of robots in $F$-formation. Let us consider the following properties:

1) $\omega_{0}$ is constant and

a) if $\omega_{0}=0$, then $\theta_{0}(t)=\bar{\theta}_{0}, \forall t \geq 0$ and, $\forall i=1, \ldots, n$

$$
\theta_{i}(t) \in\left\{\bar{\theta}_{0}, \bar{\theta}_{0}+\pi\right\}, \quad \forall t \geq 0 .
$$

b) if $\omega_{0} \neq 0$, then there exists $(\bar{x}, \bar{y})^{T} \in \mathbb{R}^{2}$ such that $\forall t \geq 0, \forall i=0, \ldots, n$

$$
\left\langle\left(\begin{array}{l}
x_{i}(t) \\
y_{i}(t)
\end{array}\right)-\left(\begin{array}{l}
\bar{x} \\
\bar{y}
\end{array}\right), \tau\left(\theta_{i}(t)\right)\right\rangle=0
$$

2) $\llbracket \mathcal{R} \rrbracket$ is constant.

Then 1) implies 2). Vice versa, if the following property holds:

$$
\left\{\begin{array}{c}
\mathcal{F} \text { does not contain any point of the set : } \\
\Sigma=\left\{\begin{array}{c}
\xi \in \mathcal{X} \mid\left\langle\left(\begin{array}{l}
x_{i}-x_{0} \\
y_{i}-y_{0}
\end{array}\right), \tau\left(\theta_{0}\right)\right\rangle=0, \\
\left.\theta_{i} \in\left\{\theta_{0}, \theta_{0}+\pi\right\}, \forall i=1, \ldots, n\right\} .
\end{array}\right.
\end{array}\right.
$$

then 2) implies 1).

The proof is reported in the Appendix.

Definition 11: For any $i=1, \ldots, n$, let $\Theta: \mathcal{X} \rightarrow S^{1}$ be the map defined by

$$
\Theta_{i}(\xi)=\theta_{i}
$$

(recall that $\xi=\left(\xi_{0}, \xi_{1}, \ldots, \xi_{n}\right)^{T}$ and $\xi_{i}=\left(x_{i}, y_{i}, \theta_{i}\right)^{T}, \forall i=$ $1, \ldots, n)$.

Therefore, if we set the $n$-dimensional torus $T^{n}=\{\beta=$ $\left.\left(\beta_{1}, \ldots, \beta_{n}\right)^{T} \mid \beta_{i} \in S^{1}, i=1, \ldots, n\right\}$, the map $\Theta: \mathcal{X} \rightarrow T^{n}$ defined by $\Theta(\xi)=\left(\Theta_{1}(\xi), \ldots, \Theta_{n}(\xi)\right)^{T}=\left(\theta_{1}, \ldots, \theta_{n}\right)^{T}$, is the projection of $\mathcal{X}$ on the torus of the followers' heading angles.

Suppose that $F$ is a regular rototranslation invariant constraint function such that, $\forall \xi \in \Gamma_{F}$

$$
\operatorname{det}\left(\partial_{x_{1}} F(\xi), \partial_{y_{1}} F(\xi), \ldots, \partial_{x_{n}} F(\xi), \partial_{y_{n}} F(\xi)\right) \neq 0 .
$$

By the implicit function theorem, the restriction on $\Gamma_{F}$ of the projection

$$
\begin{aligned}
\Theta: \Gamma_{F} & \longrightarrow T^{n} \\
\xi & \rightsquigarrow \Theta(\xi)=\left(\theta_{1}, \ldots, \theta_{n}\right)^{T}
\end{aligned}
$$

is a local $\mathcal{C}^{1}$ diffeomorphism. Observe that, since $\xi \in \Gamma_{F}, \theta_{i}$ represents the relative heading angle between the $i$ th robot and the leader. This justifies the following definition.

Definition 12: The function $F$ is called a relative angle parametrizable constraint function if it satisfies the following properties:

1) $F$ is regular;

2) $F$ is rototranslation invariant;

3) $F$ verifies property (31).

Remark 7: Suppose that $F$ is a relative angle parametrizable constraint function. For any $\omega_{0} \in \mathcal{C}([0,+\infty[, \mathbb{R})$ set $\varphi_{\omega_{0}}:\left[0,+\infty\left[\times \Gamma_{F} \rightarrow \Gamma_{F}\right.\right.$ the map defined by $\varphi_{\omega_{0}}(t, \xi)=\mathcal{J}\left(\Phi_{\omega_{0}}(t, \llbracket \xi \rrbracket)\right)$, where $\mathcal{J}$ is given by (22). For any $\bar{\xi} \in \Gamma_{F}$, set $\bar{\beta}=\Theta(\bar{\xi}) \in T^{n}$. Since $\Theta$ is a local diffeomorphism, there exists a neighborhood $U$ of $\bar{\xi} \in \Gamma_{F}$ on which $\Theta$ is a diffeomorphism. Then the reduced internal dynamics system (28) becomes (in the torus variables of $\Theta(U)$ )

$$
\left\{\begin{array}{l}
\dot{\beta}=\psi\left(\beta(t), \omega_{0}(t)\right) \\
\beta(0)=\bar{\beta}
\end{array}\right.
$$




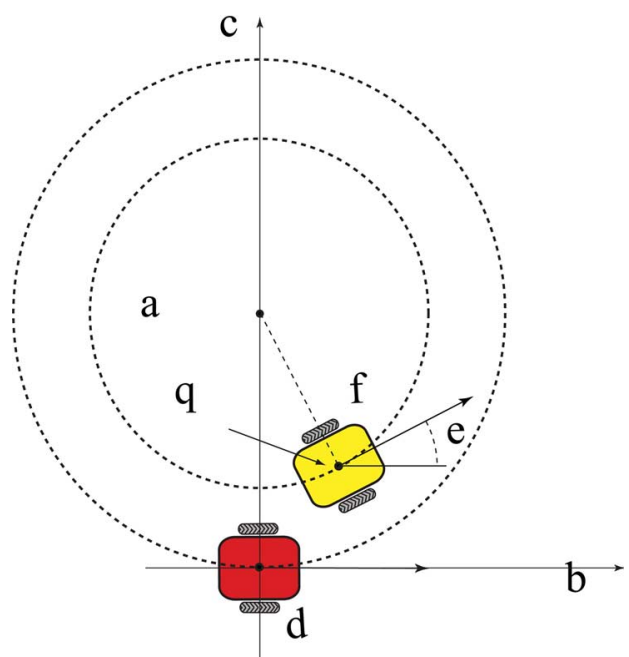

Fig. 6. Illustration of the property satisfied by $\bar{\xi}$, solution of (35), in Lemma 1.

where $\omega_{0} \in \mathcal{C}^{0}([0,+\infty[, \mathbb{R})$ and $\forall \beta \in \Theta(U)$

$$
\psi\left(\beta, \omega_{0}\right)=\left.\frac{d}{d t}\left(\varphi_{\omega_{0}}\left(t, \Theta^{-1}(\beta)\right)\right)\right|_{t=0}
$$

Therefore, $\forall\left(\beta, \omega_{0}\right) \in \Theta(U) \times \mathbb{R}, \psi\left(\beta, \omega_{0}\right)=0$ if and only if there exists an $(n+1)$-tuple of robots $\mathcal{R}$, which is in $F$-formation (with leader's control $\left(1, \omega_{0}\right)$ ) and is such that $\llbracket \mathcal{R}(t) \rrbracket=\Theta^{-1}(\beta), \forall t \geq 0$, i.e., the reduced motion of $\mathcal{R}$ is constant. As a consequence, if $0 \in \Theta\left(\Gamma_{F}\right), \psi(0,0)=0$, since $F$ is rototranslation invariant.

The next theorem shows that if the leader moves along a straight line or a circle with radius greater than $\rho_{F}$, then there exist trajectories for the followers such that they are in formation and their positions and orientations are fixed in the leader's reference frame. This means that the followers move along parallel straight lines or circular trajectories concentric with the one of the leader. In other words, the theorem provides a sufficient condition for the existence of an equilibrium for the reduced internal dynamics when the curvature of the path of the leader is constant and smaller in absolute value than $1 / \rho_{F}$.

Theorem 2: Let $F$ be a relative angle parameterizable constraint function and suppose that $\rho_{F}<+\infty$ (recall definition 9). Then, for any $\bar{\omega}$ such that

$$
-\frac{1}{\rho_{F}}<\bar{\omega}<\frac{1}{\rho_{F}}
$$

there exists an $(n+1)$-tuple of robots $\mathcal{R}$ such that the reduced motion $\llbracket \mathcal{R} \rrbracket$ is constant and $\omega_{0}(t)=\bar{\omega}, \forall t \geq 0$.

To prove theorem 2 we need the following lemma, whose proof is reported in the Appendix.

Lemma 1: Suppose that the hypotheses of theorem 2 hold. Then:

1) $\Theta: \Gamma_{F} \rightarrow T^{n}$ is surjective;

2) if $\bar{\omega} \neq 0$, there exists $\bar{\xi} \in \Gamma_{F}$ such that

$$
\Theta_{i}(\bar{\xi})=\arg \left(\Pi_{i}(\bar{\xi})-\left(\begin{array}{c}
0 \\
\frac{1}{\bar{\omega}}
\end{array}\right)\right)+\frac{\pi}{2}, \quad \forall i=1, \ldots, n .
$$

Proof of Theorem 2: First of all, suppose that $\bar{\omega}=0$, then by 1) of lemma $1, \Theta: \Gamma_{F} \rightarrow T^{n}$ is surjective and there exists

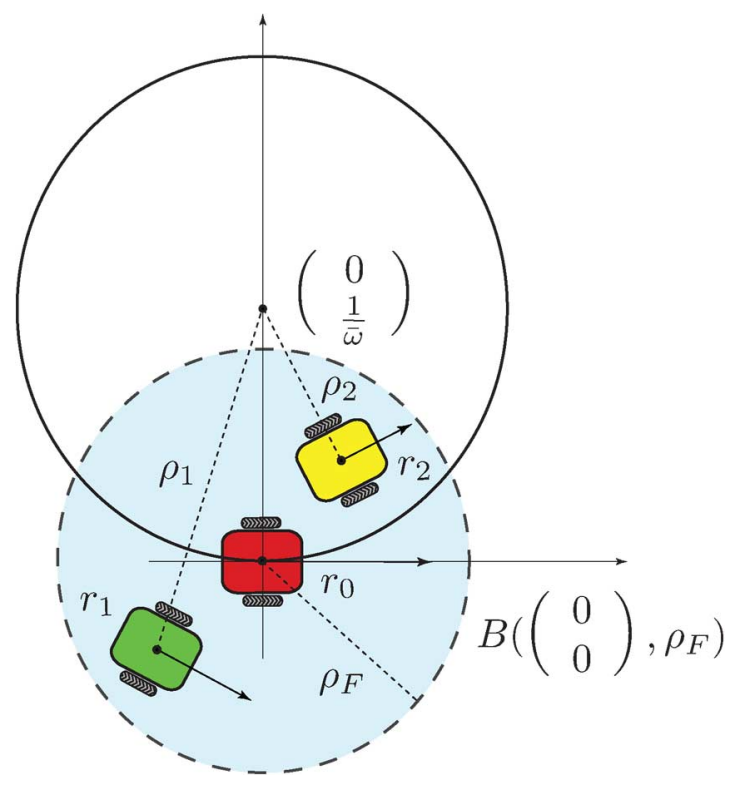

Fig. 7. Setting used in the proof of theorem 2.

$\zeta \in \Gamma_{F}$, such that $\Theta(\zeta)=0 \in T^{n}$. Define $\mathcal{R}(t)=\zeta, \forall t \geq 0$. Then $\mathcal{R}$ is an $(n+1)$-tuple of robots since it verifies system (5) with $v_{i}=1, \theta_{i}=0, \forall i=0, \ldots, n$. Moreover, $\mathcal{R}$ is in $F$-formation since $\zeta \in \Gamma_{F}$ and the reduced motion $\llbracket \mathcal{R} \rrbracket$ of $\mathcal{R}$ is constantly equal to $\zeta$ and $\omega_{0}(t)=0=\bar{\omega}, \forall t \geq 0$. Suppose now that $\bar{\omega} \neq 0$. Set $\bar{\beta}=\left(\bar{\beta}_{1}, \ldots, \bar{\beta}_{n}\right)^{T}=\Theta(\bar{\xi})$, where $\bar{\xi}$ is given by (35). Then $\bar{\beta}_{i}=\Theta_{i}(\bar{\xi})$ and by (35) (see Fig. 6)

$$
\bar{\beta}_{i}=\arg \left(\Pi_{i}(\bar{\xi})-\left(\begin{array}{c}
0 \\
\frac{1}{\bar{\omega}}
\end{array}\right)\right)+\frac{\pi}{2}, \quad \forall i=1, \ldots, n .
$$

Let $r_{0}=\left(x_{0}, y_{0}, \theta_{0}\right)^{T}$ be the leader robot which has the constant controls $v_{0}(t)=1, \omega_{0}(t)=\bar{\omega}, \forall t \geq 0$ and initial condition $x_{0}(0)=0, y_{0}(0)=0, \theta_{0}(0)=0$ and set, $\forall i=1, \ldots, n$ $r_{i}(t)=\left(x_{i}(t), y_{i}(t), \theta_{i}(t)\right)^{T}$, where

$$
\begin{aligned}
\left(\begin{array}{l}
x_{i}(t) \\
y_{i}(t)
\end{array}\right) & =\left(\begin{array}{l}
x_{0}(t) \\
y_{0}(t)
\end{array}\right)+R\left(\theta_{0}(t)\right) \Pi_{i}(\bar{\xi}) \\
\theta_{i}(t)-\theta_{0}(t) & =\bar{\beta}_{i} .
\end{aligned}
$$

First of all, $r_{i}$ are robots, $\forall i=1, \ldots, n$. In fact, by (36) it follows that

$$
\begin{aligned}
\frac{d}{d t}\left(\begin{array}{l}
x_{i}(t) \\
y_{i}(t)
\end{array}\right) & =R\left(\theta_{0}(t)\right)\left(\begin{array}{l}
1 \\
0
\end{array}\right)+\bar{\omega} R\left(\theta_{0}(t)+\frac{\pi}{2}\right) \Pi_{i}(\bar{\xi}) \\
& =\bar{\omega} R\left(\theta_{0}(t)+\frac{\pi}{2}\right)\left(\Pi_{i}(\bar{\xi})-\left(\begin{array}{l}
0 \\
\frac{1}{\bar{\omega}}
\end{array}\right)\right) \\
& =\rho_{i} \bar{\omega} R\left(\theta_{0}(t)+\frac{\pi}{2}\right) R\left(\bar{\beta}_{i}-\frac{\pi}{2}\right)\left(\begin{array}{l}
1 \\
0
\end{array}\right) \\
& =\rho_{i} \bar{\omega} R\left(\theta_{i}(t)\right)\left(\begin{array}{l}
1 \\
0
\end{array}\right)
\end{aligned}
$$

where $\rho_{i}=\left\|\Pi_{i}(\bar{\xi})-\left(\begin{array}{c}0 \\ 1 / \bar{\omega}\end{array}\right)\right\|$. Then $\mathcal{R}=\left(r_{0}, r_{1}, \ldots, r_{n}\right)$ is an $(n+1)$-tuple of robots which is in $F$-formation and $\llbracket R \rrbracket$ is constant (see Fig. 7), by remarks 6 and part 1) of proposition 3, since $H_{-r_{0}(t)}(\mathcal{R}(t))=\bar{\xi} \in \Gamma_{F}$ by construction.

Remark 8: Following the proof of lemma 1, the equilibrium point $\bar{\theta}=\left(\bar{\theta}_{1}, \ldots, \bar{\theta}_{n}\right)$ can be found by computing iteratively $n$ fixed points of a one-variable function, defined on $[-\pi / 2, \pi / 2]$, 


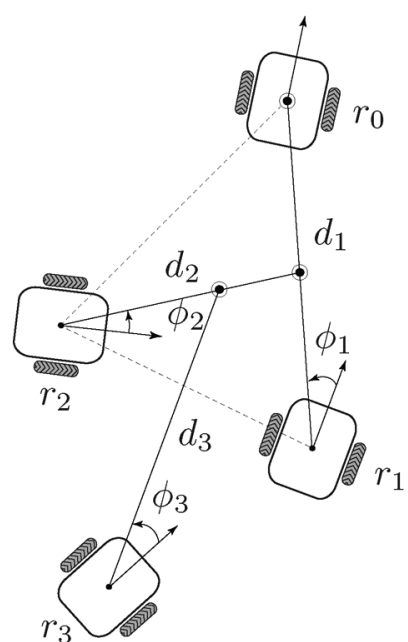

(a)

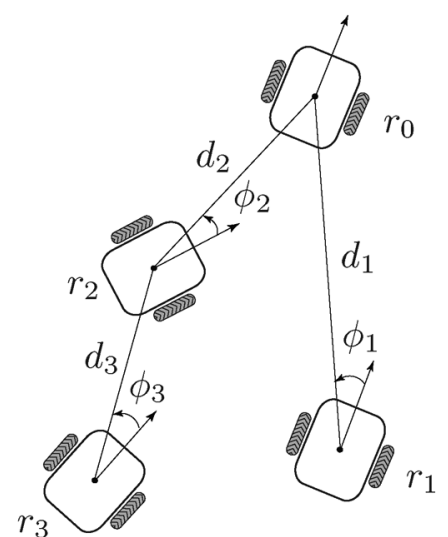

(b)

Fig. 8. (a) Sample formation of four robots, with $\lambda_{i, k}=1 / i, i=1,2,3$, $k=0, \ldots, i-1$. (b) Formation belonging to the class studied in [40].

given by (69) (see the Appendix). A root-finding algorithm for a scalar function, for example the bisection method, can be used for this purpose.

\section{Application to a Specific Hierarchical CONSTRAINT FUNCTION}

In this section, we apply the theoretical results of Section II to a specific constraint function $F$. To define $F=\left(F_{1}, \ldots, F_{n}\right)$, we assign for any $i=1, \ldots, n$ the following parameters:

$$
\lambda_{i, 0}, \ldots, \lambda_{i, i-1} \geq 0: \sum_{k=0}^{i-1} \lambda_{i, k}=1, d_{i}>0,-\frac{\pi}{2}<\phi_{i}<\frac{\pi}{2}
$$

and set

$$
F_{i}\left(\xi_{0}, \xi_{1}, \ldots, \xi_{i}\right)=\sum_{k=0}^{i-1} \lambda_{i, k}\left(\begin{array}{l}
x_{k} \\
y_{k}
\end{array}\right)-\left(\begin{array}{c}
x_{i} \\
y_{i}
\end{array}\right)-d_{i} \tau\left(\theta_{i}+\phi_{i}\right) .
$$

With this constraint function, we require robot $i$ to follow an assigned convex combination of the positions of the preceding $i-1$ robots, at a fixed distance $d_{i}$ and with a fixed visual angle $\phi_{i}$ [see Fig. 8(a)].

This particular function may be useful to describe formations occurring in nature, such as, e.g., birds flocks, where it is believed that each bird follows an average of the preceding ones [41].

Note that if, $\forall i=1, \ldots, n$, there exists an integer $l_{i}$ such that $0 \leq l_{i} \leq i-1$ and $\lambda_{i, k}=\left\{\begin{array}{ll}0, & \text { if } k \neq l_{i} \\ 1, & \text { if } k=l_{i}\end{array}\right.$ then $F$ induces the kind of "hierarchical" formations studied in [40] [see Fig. 8(b)]. In addition, if $i=1$, we end up with the leader-follower formations investigated in [39].

$F$ is a regular constraint function since (3) holds and (6) is satisfied. In fact $\left|\phi_{i}\right|<\pi / 2$ by hypothesis and $\operatorname{det}\left(\cos \theta_{i} \partial_{x_{i}} F_{i}+\right.$ $\left.\sin \theta_{i} \partial_{y_{i}} F_{i}, \partial_{\theta_{i}} F_{i}\right)=d_{i} \cos \phi_{i}$. Clearly, $F$ is rototranslation invariant, and it satisfies (30) and (31). Therefore, $F$ is a relative angle parameterizable constraint function which verifies the nonalignment condition (30) and the results developed in Section II can be applied. In particular, from proposition 1, we

have that if $\mathcal{R}$ is an $(n+1)$-tuple of robots, which is in $F$-formation at the initial time (i.e., $F(\mathcal{R}(0))=0$ ), then for any trajectory of the leader (i.e., $\forall \omega_{0} \in \mathcal{C}^{0}\left(\left[0,+\infty[, \mathbb{R})\right.\right.$, recall that $v_{0}$ is assumed to be equal to 1$)$, there exist and are unique the controls $v_{F}=\left(v_{1}, \ldots, v_{n}\right)^{T}, \omega_{F}=\left(\omega_{1}, \ldots, \omega_{n}\right)^{T}$ for the followers such that $\mathcal{R}$ is in $F$-formation for any $t \geq 0$ (i.e., $F(\mathcal{R}(t))=0$, $\forall t \geq 0$ ). These controls are given by

$$
\begin{aligned}
& v_{i}(t)=\frac{1}{\cos \phi_{i}} \sum_{h=0}^{i-1} \lambda_{i, h} v_{h}(t) \cos \left(\theta_{h}(t)-\theta_{i}(t)-\phi_{i}\right) \\
& \omega_{i}(t)=\frac{1}{d_{i} \cos \phi_{i}} \sum_{h=0}^{i-1} \lambda_{i, h} v_{h}(t) \sin \left(\theta_{h}(t)-\theta_{i}(t)\right)
\end{aligned}
$$

and the stabilizing controls [see (14)] are given by

$$
\begin{array}{r}
v_{i}(t)=\frac{1}{\cos \phi_{i}}\left(\sum_{h=0}^{i-1} \lambda_{i, h} v_{h}(t) \cos \left(\theta_{h}(t)-\theta_{i}(t)-\phi_{i}\right)\right. \\
-k \tau\left(\theta_{i}(t)+\phi_{i}\right)^{T} \\
\times\left(\sum_{h=0}^{i-1} \lambda_{i, h}\left(\begin{array}{l}
x_{h}(t) \\
y_{h}(t)
\end{array}\right)-\left(\begin{array}{l}
x_{i}(t) \\
y_{i}(t)
\end{array}\right)\right. \\
\left.\left.-d_{i} \tau\left(\theta_{i}(t)+\phi_{i}\right)\right)\right) \\
\begin{array}{r}
\omega_{i}(t)=\frac{1}{d_{i} \cos \phi_{i}}\left(\sum_{h=0}^{i-1} \lambda_{i, h} v_{h}(t) \sin \left(\theta_{h}(t)-\theta_{i}(t)\right)\right. \\
-k \nu\left(\theta_{i}(t)\right)^{T} \\
\times\left(\sum_{h=0}^{i-1} \lambda_{i, h}\left(\begin{array}{l}
x_{h}(t) \\
y_{h}(t)
\end{array}\right)-\left(\begin{array}{l}
x_{i}(t) \\
y_{i}(t)
\end{array}\right)\right. \\
\left.\left.-d_{i} \tau\left(\theta_{i}(t)+\phi_{i}\right)\right)\right)
\end{array}
\end{array}
$$

where $k$ is a positive real number. In particular, from theorem 1 we have that an $(n+1)$-tuple of robots $\mathcal{R}$ in $F$-formation has a fixed configuration in the leader's reference frame (i.e., $\llbracket \mathcal{R}(t) \rrbracket$ is constant) if and only if all robots move along straight lines or concentric circles. From theorem 2 we have that such a fixed relative configuration exists when the leader's curvature $\omega_{0}$ is constant and $\left|\omega_{0}\right|<1 / \rho_{F}$, where $\rho_{F}$ is given by definition 9 . In this specific example, $\rho_{F}$ can be bounded as follows:

$$
\rho_{F} \leq \max _{i=1, \ldots, n} \bar{\rho}_{i}
$$

where $\bar{\rho}_{0}=0$ and $\bar{\rho}_{i}=d_{i}+\sum_{k=0}^{i-1} \lambda_{i, k} \bar{\rho}_{k}$. Moreover, the $i$ th component of the reduced internal dynamics system (32) is given by

$$
\dot{\beta}_{i}(t)=\psi_{i}\left(\beta(t), \omega_{0}(t)\right), \quad i=1, \ldots, n
$$

where

$$
\begin{aligned}
& \psi_{i}\left(\beta(t), \omega_{0}(t)\right)=-\omega_{0}(t) \\
& -\frac{1}{d_{i} \cos \phi_{i}} \sum_{k=0}^{i-1} \lambda_{i, k} v_{k}(\beta) \sin \left(\beta_{i}(t)-\beta_{k}(t)\right)
\end{aligned}
$$


being

$$
v_{i}(\beta)=\frac{1}{\cos \phi_{i}} \sum_{k=0}^{i-1} \lambda_{i, k} v_{k}(\beta) \cos \left(\beta_{k}-\beta_{i}-\phi_{i}\right) .
$$

These expressions are obtained by computing the derivatives of $\beta_{i}=\theta_{i}-\theta_{0}$ and taking into account (39) (notice that $\beta_{i}$ represents the relative heading angle between the $i$ th robot and the leader). Note that, in this case, the projection $\Theta: \Gamma_{F} \rightarrow T^{n}$ is a global diffeomorphism, not only local as in the general case (see remark 7).

Remark 9: There is a strong resemblance between (42) and Kuramoto model for coupled oscillators (see for instance equation (3.1) of [33]), which has the form

$$
\dot{\theta}_{i}=\omega_{i}+\frac{K}{n} \sum_{k=1}^{n} \sin \left(\theta_{k}-\theta_{i}\right), \quad i=1, \ldots, n
$$

where $\theta_{i}$ denotes the phase of oscillator $i, \omega_{i}$ its natural frequency and $K \geq 0$ is the coupling strength.

In fact, (42) can be rewritten in terms of $\theta=\left(\theta_{1}, \ldots, \theta_{n}\right)^{T}$ in the following way:

$$
\dot{\theta}_{i}=\frac{1}{d_{i} \cos \phi_{i}} \sum_{k=0}^{i-1} \lambda_{i, k} v_{k}(\theta) \sin \left(\theta_{k}-\theta_{i}\right), \quad i=1, \ldots, n
$$

where $v_{k}(\theta)$ is given by (43).

The similarity is evident, note however the following two differences.

1) In (44) the leader's heading angle $\theta_{0}$ is an assigned function. As it will be shown in theorem 3 , the angles $\theta_{i}$, solutions of (44), follow $\theta_{0}$ by maintaining themselves at a maximum distance from it that depends on the maximum value of $\dot{\theta}_{0}$ (see property (48), below).

2) In (44), the sine function is multiplied by the velocity $v_{k}(\theta)$. Hence, in our case, the coupling strength $K$ is a nonlinear function of the oscillators' state.

Given an $(n+1)$-tuple of robots, which is in $F$-formation, the following theorem provides a method to determine an invariant region for the reduced internal dynamics (41), whose size depends on the bounds on the curvature of the path followed by the leader. In other words, this theorem allows to find a bounded region in the leader's reference frame, where the followers are confined when the team moves.

Theorem 3: Set $v_{0}=1$, let $\omega_{0}^{-} \leq \omega_{0}^{+}$be real constants and define the following constants:

$$
\beta_{0}^{-}=\beta_{0}^{+}=0
$$

$$
\begin{aligned}
& \forall i=1, \ldots, n \\
& \beta_{i}^{+}=\max \left\{\arg \left(\sum_{k=0}^{i-1} \lambda_{i, k} z_{k} e^{\beta_{k}^{+} j}\right)\right. \\
& -\arcsin \left(\frac{\omega_{0}^{+} d_{i} \cos \phi_{i}}{\left|\sum_{k=0}^{i-1} \lambda_{i, k} z_{k} e^{\beta_{k}^{+} j}\right|}\right) \\
& \left.\mid\left(z_{0}, \ldots, z_{i-1}\right) \in V_{i}\right\}
\end{aligned}
$$

$$
\begin{aligned}
& \beta_{i}^{-}=\min \left\{\arg \left(\sum_{k=0}^{i-1} \lambda_{i, k} z_{k} e^{\beta_{k}^{-} j}\right)\right. \\
&-\arcsin \left(\frac{\omega_{0}^{-} d_{i} \cos \phi_{i}}{\left|\sum_{k=0}^{i-1} \lambda_{i, k} z_{k} e^{\beta_{k}^{-} j}\right|}\right) \\
&\left.\mid\left(z_{0}, \ldots, z_{i-1}\right) \in V_{i}\right\}
\end{aligned}
$$

where $e^{\alpha j}=\cos \alpha+j \sin \alpha, \forall \alpha \in S^{1}$ and $V_{i}=\{1\} \times$ $\left\{v_{1}^{-}, v_{1}^{+}\right\} \times \cdots \times\left\{v_{i-1}^{-}, v_{i-1}^{+}\right\}$

$$
\begin{aligned}
& v_{0}^{-}=v_{0}^{+}=1, \\
& v_{i}^{-}=\frac{1}{\cos \phi_{i}} \sum_{k=0}^{i-1} \lambda_{i, k} v_{k}^{-} \cos \left(\operatorname { m a x } \left\{\left|\phi_{i}-\left(\beta_{k}^{+}-\beta_{i}^{-}\right)\right|\right.\right. \\
& v_{i}^{+}=\frac{1}{\cos \phi_{i}} \sum_{k=0}^{i-1} \lambda_{i, k} v_{k}^{+} \cos a_{i k}
\end{aligned}
$$

where

$$
a_{i k}=\left\{\begin{array}{c}
0, \text { if } \phi_{i}-\left(\beta_{k}^{+}-\beta_{i}^{-}\right) \leq 0 \leq \phi_{i}-\left(\beta_{k}^{-}-\beta_{i}^{+}\right) \\
\min \left\{\left|\phi_{i}-\left(\beta_{k}^{+}-\beta_{i}^{-}\right)\right|,\right. \\
\left.\left|\phi_{i}-\left(\beta_{k}^{-}-\beta_{i}^{+}\right)\right|\right\}, \text {otherwise. }
\end{array}\right.
$$

Set $\mathcal{B}=\left[\beta_{1}^{-}, \beta_{1}^{+}\right] \times \cdots \times\left[\beta_{n}^{-}, \beta_{n}^{+}\right]$. If

$$
v_{i}^{-} \geq 0,\left|\beta_{i}^{ \pm}\right|<\frac{\pi}{4}, \forall i=1, \ldots, n
$$

and $\omega_{0} \in \mathcal{C}^{0}([0,+\infty[, \mathbb{R})$ is such that

$$
\omega_{0}^{-} \leq \omega_{0}(t) \leq \omega_{0}^{+}
$$

then the following invariance property holds:

If $\beta$ is the solution of (41) with $\beta(0) \in \mathcal{B}$, then

$$
\beta(t) \in \mathcal{B}, \forall t \geq 0
$$

and, $\forall i=1, \ldots, n$, the functions $v_{i}$ given by (43), are such that

$$
v_{i}^{-} \leq v_{i}(t) \leq v_{i}^{+}, \forall t \geq 0 .
$$

Remark 10:

1) Property (48) guarantees that, during the motion, the relative orientation between each follower and the leader is bounded by constants $\beta_{i}^{ \pm}$. This condition limits the possible variations of the formation shape in the leader's reference frame. Once the weights $\lambda_{i, k}$ have been assigned in function (38), one can compute $\beta_{i}^{-}, \beta_{i}^{+}, v_{i}^{-}, v_{i}^{+}$for all values of $\omega_{0}^{-}$and $\omega_{0}^{+}$for which (46) is satisfied. In this way it is possible to obtain bounds on the followers' velocities and on their misalignment with respect to the leader, for different choices of the maximum curvature allowed to the leader's trajectory.

2) There always exist (sufficiently small) values of positive and negative curvature for the path of the leader such that the hypotheses of theorem 3 are verified and condition (48) holds. In fact, since condition (46) of theorem 3 holds when $\omega_{0}^{-}=\omega_{0}^{+}=0$, by continuity, there exists a real constant 


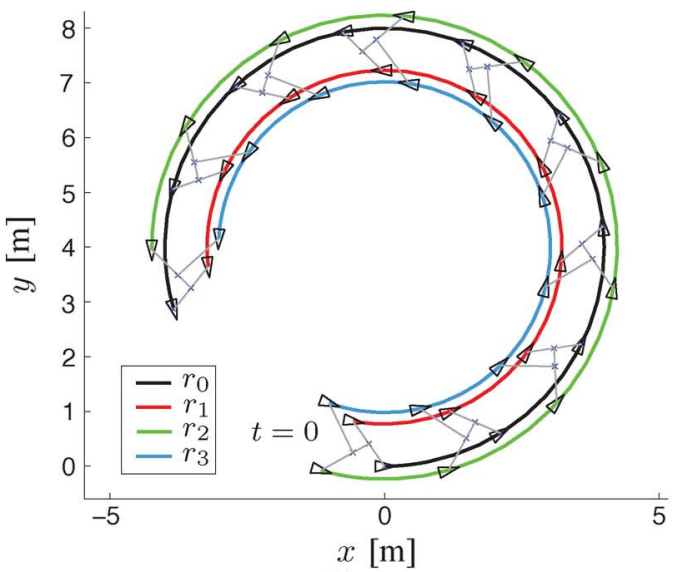

(a)

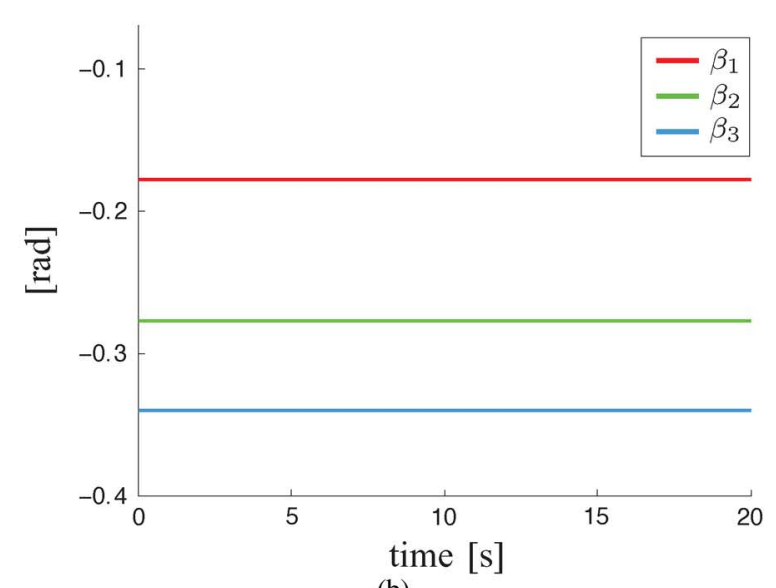

(b)

Fig. 9. First simulation: (a) Trajectory of the four robots. (b) Time history of $\beta_{i}$.

$\epsilon>0$ such that, if $\omega_{0}^{-}=-\epsilon$ and $\omega_{0}^{+}=\epsilon$, condition (46) is still satisfied.

Proof of Theorem 3: Let $\omega_{0} \in \mathcal{C}([0,+\infty[, \mathbb{R})$ be such that (48) holds and $\beta \in \mathcal{C}^{1}\left(\left[0,+\infty\left[, \mathbb{R}^{n}\right)\right.\right.$ be a solution of the reduced internal dynamics system (41) such that $\beta(0) \in \mathcal{B}$. Note that (48) and (49) hold if we show that the following property is true (recall that $v_{0}(t)=1, \forall t \geq 0$ ):

$$
\left\{\begin{array}{l}
\text { Let } i \text { be such that } 1<i \leq n . \\
\text { If } \forall k=0, \ldots, i-1, \forall t \geq 0, \\
\quad \beta_{k}^{-} \leq \beta_{k}(t) \leq \beta_{k}^{+}, v_{k}^{-} \leq v_{k}(t) \leq v_{k}^{+}, \\
\text {then, } \forall t \geq 0 \\
\beta_{i}^{-} \leq \beta_{i}(t) \leq \beta_{i}^{+}, \\
v_{i}^{-} \leq v_{i}(t) \leq v_{i}^{+} .
\end{array}\right.
$$

Observe that, by (50)

$$
\left(v_{1}(t), \ldots, v_{i-1}(t)\right)^{T} \in\left[v_{1}^{-}, v_{1}^{+}\right] \times \cdots \times\left[v_{i-1}^{-}, v_{i-1}^{+}\right] .
$$

Note that $V_{i}$ represents the set of the vertices of the polyhedron $\{1\} \times\left[v_{i}^{-}, v_{i}^{+}\right] \times \cdots \times\left[v_{i-1}^{-}, v_{i-1}^{+}\right]$. The cardinality of $V_{i}$ is $2^{i-1}$ and its generic element is denoted by $z=\left(z_{0}, \ldots, z_{i-1}\right)$. Then, if (50) is verified, there exist $2^{i-1}$ nonnegative continuous functions $\left\{\mu_{z}(t)\right\}_{z \in V_{i}}$ such that

$$
\sum_{z \in V_{i}} \mu_{z}=1 \text { and }\left(1, v_{1}(t), \ldots, v_{i-1}(t)\right)^{T}=\sum_{z \in V_{i}} \mu_{z}(t) z
$$

Therefore,

$$
\begin{aligned}
\psi_{i}\left(\beta(t), \omega_{0}\right)= & -\omega_{0}(t)-\sum_{k=0}^{i-1} \lambda_{i, k}\left(\sum_{z \in V_{i}} \mu_{z}(t) z\right)_{k} \\
& \times \sin \left(\beta_{i}(t)-\beta_{k}(t)\right) \\
= & \sum_{z \in V_{i}} \mu_{z}(t) \psi_{i, z}\left(\beta(t), \omega_{0}(t)\right)
\end{aligned}
$$

where $\forall z \in V_{i}$

$$
\psi_{i, z}\left(\beta, \omega_{0}\right)=-\omega_{0}-\sum_{k=0}^{i-1} \lambda_{i, k} z_{k} \sin \left(\beta_{i}-\beta_{k}\right) .
$$

Set

$$
\begin{aligned}
& S_{+}=\left[\beta_{1}^{-}, \beta_{1}^{+}\right] \times \cdots \times\left[\beta_{i-1}^{-}, \beta_{i-1}^{+}\right] \times\left\{\beta_{i}^{+}\right\} \\
& S_{-}=\left[\beta_{1}^{-}, \beta_{1}^{+}\right] \times \cdots \times\left[\beta_{i-1}^{-}, \beta_{i-1}^{+}\right] \times\left\{\beta_{i}^{-}\right\}
\end{aligned}
$$

then by definition (45) we get that $\forall z \in V_{i}$ :

$$
\begin{aligned}
& \psi_{i, z}\left(\beta, \omega_{0}\right) \leq 0, \forall \beta \in S_{+}, \forall \omega_{0} \in\left[\omega_{0}^{-}, \omega_{0}^{+}\right] \\
& \psi_{i, z}\left(\beta, \omega_{0}\right) \geq 0, \forall \beta \in S_{-}, \forall \omega_{0} \in\left[\omega_{0}^{-}, \omega_{0}^{+}\right]
\end{aligned}
$$

which implies, by (53), that $\forall \omega_{0} \in\left[\omega_{0}^{-}, \omega_{0}^{+}\right]$

$$
\begin{aligned}
& \psi_{i}\left(\beta, \omega_{0}\right) \leq 0, \forall \beta \in S_{+} \\
& \psi_{i}\left(\beta, \omega_{0}\right) \geq 0, \forall \beta \in S_{-}
\end{aligned}
$$

and (51) holds. Finally (52) is true by direct computation.

\section{Simulation Results}

In the simulations described in this section, we have considered the constraint function (38) with $\left(d_{1}, \phi_{1}\right)=\left(d_{2}, \phi_{2}\right)=$ $\left(d_{3}, \phi_{3}\right)=(1, \pi / 4)$. We set $\lambda_{i, k}=1 / i, i=1,2,3, k=$ $0, \ldots, i-1$ : in this way robot $i$ follows exactly the average of the positions of the preceding $i-1$ robots in the formation. Applying (40), we have that $1 / \rho_{F} \geq 0.546$. Let $\bar{\omega}=0.25$, then, by theorem 2 , there exists an equilibrium configuration $\bar{\beta}$ for which $\omega_{0}(t)=0.25, \forall t \geq 0$. It can be obtained solving iteratively the fixed-point (36), or, equivalently, it is such that $\psi(\bar{\beta}, \bar{\omega})=0$, in (33). In this example, it corresponds to a solution of $\psi_{i}(\beta, \bar{\omega})=0$ for $i=1,2,3$, where $\psi_{i}$ is reported in (42). An equilibrium configuration is given by $\bar{\beta}=\left(\bar{\beta}_{1}, \bar{\beta}_{2}, \bar{\beta}_{3}\right)^{T}=$ $(-0.177,-0.277,-0.339)^{T}$. Hence, if at the initial time the robots are in formation and $\beta(0)=\bar{\beta}$, the positions of the followers are fixed in the leader's reference frame [that is $\beta(t)=\bar{\beta}$, $\forall t \geq 0$, see Fig. 9(b)] and they move along concentric circles [see Fig. 9(a)].

Let us now suppose that $\omega_{0}(t)=0.25 \sin (t / 4)$, then $\omega_{0}^{-}(t)=$ $-0.25 \leq \omega_{0}(t) \leq \omega_{0}^{+}=0.25, \forall t \geq 0$. By means of (45) we see that $\beta_{1}^{ \pm}= \pm 0.177, \beta_{2}^{ \pm}= \pm 0.277, \beta_{3}^{ \pm}= \pm 0.349$; 


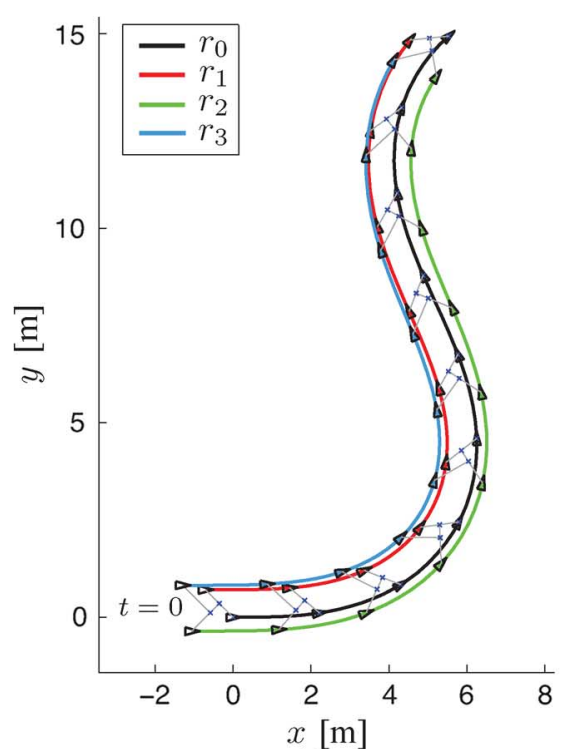

(a)

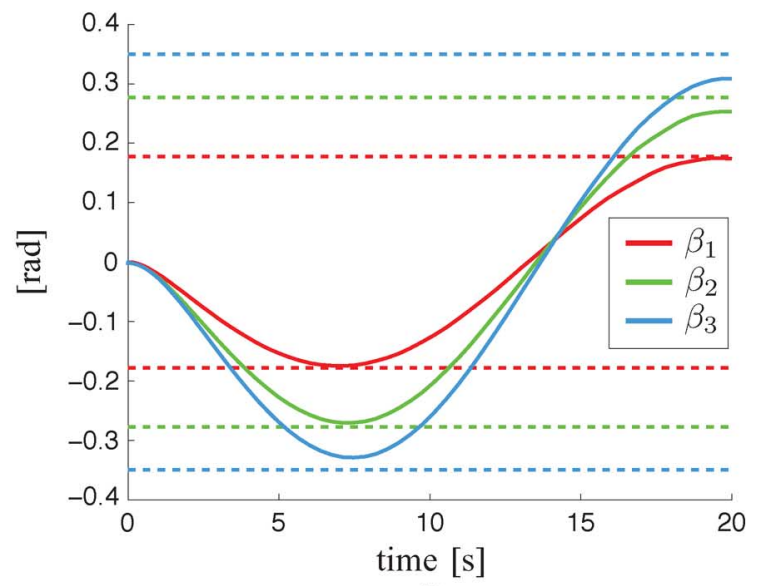

(b)

Fig. 10. Second simulation: (a) Trajectory of the four robots. (b) Time history of $\beta_{i}$ (solid) and bounds $\beta_{i}^{ \pm}$(dash).

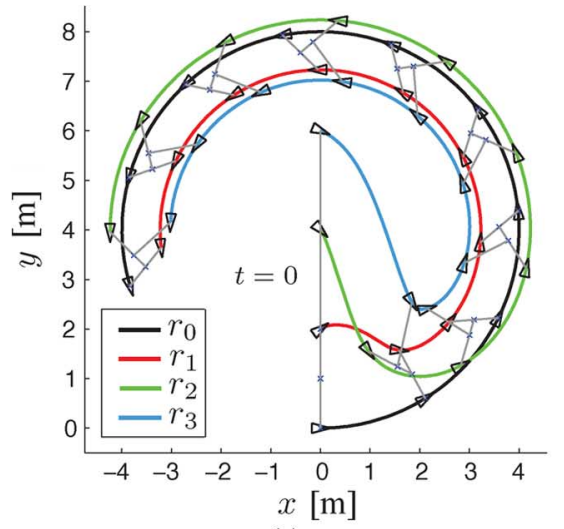

(a)

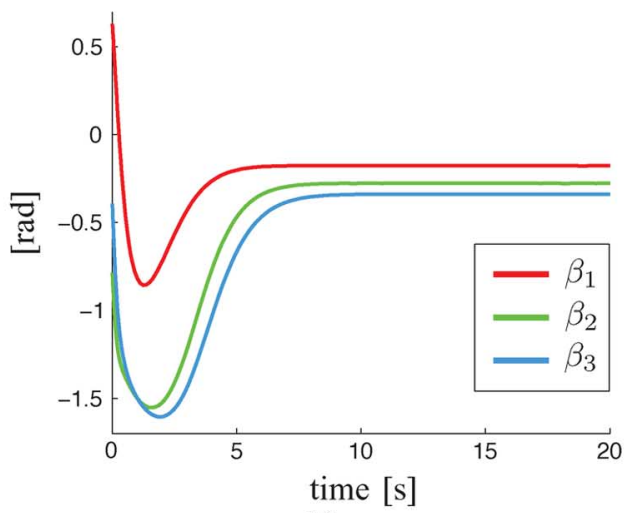

(b)

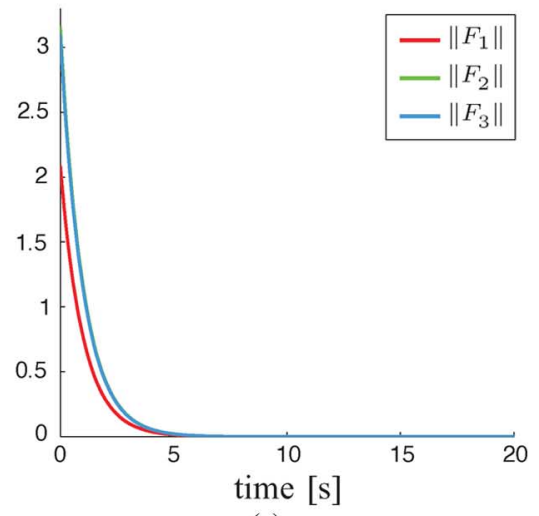

(c)

Fig. 11. Third simulation, stabilization: (a) Trajectory of the four robots. (b) Time history of $\beta_{i}$. (c) Norm of $F_{i}$.

therefore, since $v_{i}^{-} \geq 0, i=1,2,3$, by theorem 3 we have that the following invariance property holds, $\forall t \geq 0, \forall i=1,2,3$,

$$
\text { if } \beta_{i}^{-} \leq \beta_{i}(0) \leq \beta_{i}^{+} \text {then } \beta_{i}^{-} \leq \beta_{i}(t) \leq \beta_{i}^{+} .
$$

This is shown in Fig. 10(a) and (b).

Finally, let us suppose again that $\omega_{0}(t)=\bar{\omega}=0.25$ but that the robots are not initially in formation. Fig. 11(a) and (c) shows the performance of the stabilizing controller (13), with control gain $k=1$. The robots are asymptotically in $F$-formation. Moreover as we can see in Fig. 11(b), $\lim _{t \rightarrow+\infty} \beta(t)=\bar{\beta}$. This is because $\bar{\beta}$ is an equilibrium point which is asymptotically stable (the robots asymptotically move along a circle): this property can be easily verified from (41).

\section{CONCLUSION}

In this paper, we have studied a class of hierarchical formations of unicycle robots. One of the robots plays the role of the leader and the formation is induced through a constraint function $F$, which depends on the position and the orientation of the robots. We have investigated the dynamics of the formation with respect to the leader's reference frame by introducing the concept of reduced internal dynamics of the formation. We have characterized its equilibria and given sufficient conditions for their existence (theorems 1 and 2). The proposed theoretical results have been applied to the case in which the constraint $F$ induces a formation where the $i$ th robot follows a convex combination of the positions of the preceding $i-1$ robots. In this setting, we have proved an invariance property (theorem 3 ) which states that, if the curvature of the leader's trajectory is sufficiently small, the positions and orientations of the robots, relative to the leader's reference frame, are confined in a precise polyhedral region.

\section{APPENDIX}

\section{A. Proof of Theorem 1}

First part of the proof: 1) $\Rightarrow 2$ ): Suppose that a) holds. Then $\theta_{0}(t)=\bar{\theta}_{0}, \omega_{0}(t)=0, \forall t \geq 0$ and $\theta_{i}(t) \in\left\{\bar{\theta}_{0}, \bar{\theta}_{0}+\pi\right\}$, $\forall i=1, \ldots, n$. First, we show that, $\forall i=1, \ldots, n$ :

$$
v_{i}= \begin{cases}1 & \text { if } \theta_{i}(t)=\bar{\theta}_{0} \\ -1 & \text { if } \theta_{i}(t)=\bar{\theta}_{0}+\pi .\end{cases}
$$


In fact, since $\mathcal{R}$ is in $F$-formation:

$$
F\left(r_{0}(t), \ldots, r_{n}(t)\right)=0, \forall t \geq 0
$$

and, since $F$ is rototranslation invariant, $\forall t \geq 0, \forall h \in \mathbb{R}$ :

$$
h \rightsquigarrow F\left(r_{0}(t)+\left(\begin{array}{c}
\tau\left(\theta_{0}\right) \\
0
\end{array}\right) h, \ldots, r_{n}(t)+\left(\begin{array}{c}
\tau\left(\theta_{0}\right) \\
0
\end{array}\right) h\right)=0 .
$$

Then, differentiating with respect to $h$, we get for $h=0$

$$
\sum_{i=1}^{n} \partial_{\xi_{i}} F(\mathcal{R}(t))\left(\begin{array}{c}
\tau\left(\theta_{0}\right) \\
0
\end{array}\right)=-\partial_{\xi_{0}} F(\mathcal{R}(t))\left(\begin{array}{c}
\tau\left(\theta_{0}\right) \\
0
\end{array}\right) .
$$

Differentiating (55) with respect to $t$ we have also

$$
\sum_{i=1}^{n} \partial_{\xi_{i}} F(\mathcal{R}(t))\left(\begin{array}{c}
v_{i} \tau\left(\theta_{i}\right) \\
0
\end{array}\right)=-\partial_{\xi_{0}} F(\mathcal{R}(t))\left(\begin{array}{c}
\tau\left(\theta_{0}\right) \\
0
\end{array}\right)
$$

since $v_{0}=1$ by assumption 1 and $r_{i}(t)=\left(\begin{array}{c}v_{i} \tau\left(\theta_{i}\right) \\ 0\end{array}\right)$, being $\omega_{i}=0, \forall i=1, \ldots, n$. Because $F$ is regular, $v_{i}$ must satisfy (54). To prove that $\llbracket \mathcal{R} \rrbracket$ is constant, by 1 ) of proposition 3 , it is sufficient to observe that

$$
\begin{aligned}
\left.\frac{d}{d t}\left(R\left(-\theta_{0}(t)\right)\left(\left(\begin{array}{l}
x_{i}(t) \\
y_{i}(t)
\end{array}\right)-\left(\begin{array}{l}
x_{0}(t) \\
y_{0}(t)
\end{array}\right)\right)\right)\right) \\
=\omega_{0}\left(R\left(-\theta_{0}(t)+\frac{\pi}{2}\right)\left(\left(\begin{array}{l}
x_{i}(t) \\
y_{i}(t)
\end{array}\right)-\left(\begin{array}{l}
x_{0}(t) \\
y_{0}(t)
\end{array}\right)\right)\right. \\
+R\left(-\theta_{0}(t)\right)\left(v_{i} \tau\left(\theta_{i}(t)\right)-\tau\left(\theta_{0}(t)\right)\right)=0
\end{aligned}
$$

since $\omega_{0}=0, \theta_{i}(t) \in\left\{\bar{\theta}_{0}, \bar{\theta}_{0}+\pi\right\}, \forall i=1, \ldots, n$ and (54) holds.

Let us now suppose that (b) holds, then $\omega_{0}$ is a nonzero constant. By (29) it follows that

$$
\left(\begin{array}{l}
x_{i}(t) \\
y_{i}(t)
\end{array}\right)-\left(\begin{array}{l}
\bar{x} \\
\bar{y}
\end{array}\right)=\rho_{i}(t) \eta\left(\theta_{i}(t)\right), \forall t \geq 0
$$

First of all we want to show that

$$
\rho_{i}(t) \text { is constant, } \forall t \geq 0
$$

and

$$
\omega_{i}(t)=\omega_{0}, \forall t \geq 0 \text {. }
$$

In fact, (56) holds, since

$$
\begin{aligned}
\frac{d}{d t}\left(\rho_{i}^{2}(t)\right) & =\frac{d}{d t}\left(\left\|\left(\begin{array}{l}
x_{i}(t) \\
y_{i}(t)
\end{array}\right)-\left(\begin{array}{l}
\bar{x} \\
\bar{y}
\end{array}\right)\right\|^{2}\right) \\
& =2\left\langle\left(\begin{array}{l}
x_{i}(t) \\
y_{i}(t)
\end{array}\right)-\left(\begin{array}{l}
\bar{x} \\
\bar{y}
\end{array}\right), \tau\left(\theta_{i}(t)\right)\right\rangle=0
\end{aligned}
$$

by (29). To show (57), set $\forall s \in \mathbb{R}$

$$
p(s)=\left(R\left(-s \omega_{0}\right)\left(\begin{array}{l}
\bar{x} \\
\bar{y}
\end{array}\right)-\left(\begin{array}{l}
\bar{x} \\
\bar{y}
\end{array}\right), s \omega_{0}\right) .
$$

Since $F$ is rototranslation invariant and $\mathcal{R}$ is in $F$-formation, it follows that, $\forall t \geq 0, \forall s \in \mathbb{R}$ :

$$
s \rightsquigarrow F\left(H_{p(s)}(\mathcal{R}(t))\right)=0 .
$$

Note that $\forall(x, y, \theta) \in \mathbb{R}^{2} \times S^{1}$ [see (16)]

$h_{p(s)}(x, y, \theta)=\left(R\left(s \omega_{0}\right)\left(\left(\begin{array}{l}x \\ y\end{array}\right)-\left(\begin{array}{l}\bar{x} \\ \bar{y}\end{array}\right)\right)+\left(\begin{array}{l}\bar{x} \\ \bar{y}\end{array}\right), \theta+s \omega_{0}\right)$

and

$$
\begin{aligned}
& \left.\left(\frac{d}{d s} h_{p(s)}(x, y, \theta)\right)\right|_{s=0} \\
& =\left(\begin{array}{c}
\omega_{0}\left(\begin{array}{cc}
0 & -1 \\
1 & 0
\end{array}\right)\left(\left(\begin{array}{l}
x \\
y
\end{array}\right)-\left(\begin{array}{l}
\bar{x} \\
\bar{y}
\end{array}\right)\right) \\
\omega_{0}
\end{array}\right) \\
& =\left(\begin{array}{c}
\omega_{0}\left(\begin{array}{l}
x-\bar{x} \\
y-\bar{y}
\end{array}\right)^{\perp} \\
\omega_{0}
\end{array}\right)
\end{aligned}
$$

where $\left(\begin{array}{c}x-\bar{x} \\ y-\bar{y}\end{array}\right) \perp$ denotes the orthogonal of $\left(\begin{array}{c}x-\bar{x} \\ y-\bar{y}\end{array}\right)$. Then, by taking the derivative of (58) with respect to $s$, computed for $s=0$, we obtain

$$
\sum_{i=1}^{n} \partial_{\xi_{i}} F(\mathcal{R}(t))\left(\begin{array}{c}
\omega_{0} \rho_{i} \tau\left(\theta_{i}(t)\right) \\
\omega_{0}
\end{array}\right)=-\partial_{\xi_{0}} F(\mathcal{R}(t))\left(\begin{array}{c}
\tau\left(\theta_{0}\right) \\
\omega_{0}
\end{array}\right) .
$$

Since $\mathcal{R}$ is in $F$-formation, $F(\mathcal{R}(t))=0, \forall t \geq 0$. Differentiating with respect to $t,\left(v_{0}=1\right)$, we get $\forall t \geq 0$

$$
\sum_{i=1}^{n} \partial_{\xi_{i}} F(\mathcal{R}(t))\left(\begin{array}{c}
v_{i} \tau\left(\theta_{i}(t)\right) \\
\omega_{i}(t)
\end{array}\right)=-\partial_{\xi_{0}} F(\mathcal{R}(t))\left(\begin{array}{c}
\tau\left(\theta_{0}(t)\right) \\
\omega_{0}
\end{array}\right) .
$$

Since $F$ is regular, by (60) and (61) it follows that $\omega_{i}(t)=\omega_{0}$, $v_{i}(t)=\rho_{i} \omega_{0}, \forall t \geq 0$, then (57) holds, which implies in particular that there exist $\bar{\theta}_{1}, \ldots, \bar{\theta}_{n} \in S^{1}$ such that $\forall i=1, \ldots, n$

$$
\theta_{i}(t)-\theta_{0}(t)=\bar{\theta}_{i}, \forall t \geq 0 \text {. }
$$

Now we obtain that $\forall i=1, \ldots, n$

$$
\begin{aligned}
R\left(-\theta_{0}(t)\right)\left(\left(\begin{array}{l}
x_{i}(t) \\
y_{i}(t)
\end{array}\right)-\left(\begin{array}{l}
\bar{x} \\
\bar{y}
\end{array}\right)\right) & =\rho_{i}\left(\begin{array}{l}
\tau^{T}\left(\theta_{0}\right) \\
\eta^{T}\left(\theta_{0}\right)
\end{array}\right) \eta\left(\theta_{i}\right) \\
& =\rho_{i}\left(\begin{array}{c}
\sin \left(\theta_{0}-\theta_{i}\right) \\
\cos \left(\theta_{0}-\theta_{i}\right)
\end{array}\right)
\end{aligned}
$$

which is constant by (56) and (62). Then $\forall i=1, \ldots, n, \forall t \geq 0$ :

$$
\begin{aligned}
R & \left(-\theta_{0}(t)\right)\left(\left(\begin{array}{l}
x_{i}(t) \\
y_{i}(t)
\end{array}\right)-\left(\begin{array}{l}
x_{0}(t) \\
y_{0}(t)
\end{array}\right)\right) \\
& =R\left(-\theta_{0}(t)\right)\left(\begin{array}{l}
x_{i}(t)-\bar{x} \\
y_{i}(t)-\bar{y}
\end{array}\right)-R\left(-\theta_{0}(t)\right)\left(\begin{array}{l}
x_{0}(t)-\bar{x} \\
y_{0}(t)-\bar{y}
\end{array}\right) \\
& =\bar{q}_{i} \in \mathbb{R}^{2}
\end{aligned}
$$

which implies that $\forall t \geq 0$

$$
H_{-r_{0}(t)}(\mathcal{R}(t))=\left((0,0),\left(\bar{q}_{1}, \bar{\theta}_{1}\right), \ldots,\left(\bar{q}_{n}, \bar{\theta}_{n}\right)\right)^{T}=\bar{\xi}
$$

then 1) holds by remark 5.

Second Part of the Proof: 2) plus condition $(30) \Rightarrow 1)$ : By 1) of proposition 3 and definition 6 , there exist $\bar{\theta}=\left(\bar{\theta}_{0}, \ldots, \bar{\theta}_{n}\right)^{T}$, $\bar{P}_{1}, \ldots, \bar{P}_{n} \in \mathbb{R}^{2}$ such that, $\forall i=1, \ldots, n, \forall t \geq 0$

$$
\begin{aligned}
\theta_{i}(t)-\theta_{0}(t) & =\bar{\theta}_{i}, \\
R\left(-\theta_{0}(t)\right)\left(P_{i}(t)-P_{0}(t)\right) & =\bar{P}_{i}
\end{aligned}
$$

where $P_{i}(t)=\left(\begin{array}{l}x_{i}(t) \\ y_{i}(t)\end{array}\right), \forall i=1, \ldots, n$. 
Differentiating (64), recalling that $v_{0}=1$, we obtain that

$$
R\left(-\theta_{0}\right)\left(v_{i} \tau\left(\theta_{i}\right)-\tau\left(\theta_{0}\right)\right)-\omega_{0} R\left(-\theta_{0}+\frac{\pi}{2}\right)\left(P_{i}-P_{0}\right)=0
$$

that is

$$
v_{i} \tau\left(\theta_{i}-\theta_{0}\right)-\tau(0)-\omega_{0} R\left(-\theta_{0}+\frac{\pi}{2}\right)\left(P_{i}-P_{0}\right)=0 .
$$

By left multiplying this expression by $R\left(\theta_{0}-\theta_{i}\right)$ it follows that $\forall i=1, \ldots, n$ :

$$
v_{i} \tau(0)-\tau\left(\theta_{0}-\theta_{i}\right)-\omega_{0} R\left(-\theta_{i}+\frac{\pi}{2}\right)\left(P_{i}-P_{0}\right)=0
$$

whose second component is

$$
\sin \left(\theta_{i}-\theta_{0}\right)-\omega_{0}\left\langle\tau\left(\theta_{i}\right), P_{i}-P_{0}\right\rangle=0
$$

Note that

$$
\left\langle\tau\left(\theta_{i}\right), P_{i}-P_{0}\right\rangle \neq 0, \forall i=1, \ldots, n .
$$

In fact, if this were not true, it should be (for some $t$ )

$$
\left\langle\tau\left(\theta_{i}\right), P_{i}-P_{0}\right\rangle=0 \text { and } \theta_{i} \in\left\{\theta_{0}, \theta_{0}+\pi\right\}
$$

since $\sin \left(\theta_{i}-\theta_{0}\right)=0$ by (65) which is impossible by hypothesis (30). Moreover,

$$
\left\langle\tau\left(\theta_{i}\right), P_{i}-P_{0}\right\rangle \text { is constant }
$$

by (63), (64), and because it is the second component of $R\left(\theta_{0}-\right.$ $\left.\theta_{i}\right) R\left(-\theta_{0}\right)\left(P_{i}-P_{0}\right)$. Therefore, by (63), (65), (66), and (67) we have that $\omega_{0}$ is constant. Since $\sin \left(\theta_{i}-\theta_{0}\right)=\left\langle\tau\left(\theta_{i}\right), \eta\left(\theta_{0}\right)\right\rangle$, we have, by (65)

$$
\left\langle\tau\left(\theta_{i}\right), \eta\left(\theta_{0}\right)+\omega_{0}\left(P_{i}-P_{0}\right)\right\rangle=0 .
$$

Then, if $\omega_{0} \neq 0$ it follows that $\left\langle\tau\left(\theta_{i}\right), P_{i}-\left(P_{0}+\right.\right.$ $\left.\left.\left(1 / \omega_{0}\right) \eta\left(\theta_{0}\right)\right)\right\rangle=0$. Since $P_{0}+\left(1 / \omega_{0}\right) \eta\left(\theta_{0}\right)$ is constant, being $\left.(d / d t)\left(P_{0}+\left(1 / \omega_{0}\right) \eta\left(\theta_{0}\right)\right)=0,2\right)$ is verified setting $\left(\begin{array}{l}\bar{x} \\ \bar{y}\end{array}\right)=P_{0}+\left(1 / \omega_{0}\right) \eta\left(\theta_{0}\right)$. If $\omega_{0}=0$, we obtain 1$)$ by (68).

Proof of Lemma 1: Recall that $\Theta: \Gamma_{F} \rightarrow T^{n}$, is defined by $\Theta(\xi)=\left(\theta_{1}, \ldots, \theta_{n}\right)^{T}$, which is the projection of $\Gamma_{F}$ on the torus (where $\xi=\left(0, \xi_{1}, \ldots, \xi_{n}\right) \in \Gamma_{F}$ and $\xi_{i}=\left(x_{i}, y_{i}, \theta_{i}\right)^{T}$, $\forall i=1, \ldots, n)$. Note that $\Theta$ satisfies the following properties:

1) $\Theta$ is proper, that is $\Theta^{-1}(K)$ is compact for any compact $K \subset T^{n}$. In fact if $K$ is compact in $T^{n}, \Theta^{-1}(K)$ is closed by the continuity of $\Theta$ (since $\Theta$ is a projection); hence, $\Theta^{-1}(K)$ is compact since $\Theta^{-1}(K)$ is bounded being a subset of $\Gamma_{F}$ which is contained in $\left.\left(B\left(\left(\begin{array}{l}0 \\ 0\end{array}\right), \rho_{F}\right)\right) \times S^{1}\right)^{n}$, (note that $\rho_{F}<+\infty$ by hypothesis).

2) $\Theta$ is a local diffeomorphism by (31) and the implicit function theorem. Therefore, the couple $\left(\Gamma_{F}, \Theta\right)$ is a covering map of $T^{n}$ by proposition 2.19 of [42], which implies that $\Theta$ is surjective (therefore 1) holds) and has the unique path lifting property by proposition 6.7 of [43]. Since $\mathcal{F}_{r}$ is not empty by (2), $\Gamma_{F}$ is not empty. Set $\left.D=\right]-2 \pi / 3,2 \pi / 3\left[{ }^{n} \subset\right.$ $T^{n}$ and consider $\Theta^{-1}(D)$ which is non-empty since $\Theta$ is surjective being a covering map. Let $\Theta^{-1}(D)$ be a connected component of $\Theta^{-1}(D)$. Let $\tilde{\Theta}: \Theta^{-1}(D) \rightarrow D$ be the restriction of $\Theta$ to $\Theta^{-1}(D)$, then $\tilde{\Theta}$ has the unique path lifting property (see for instance [43, Sec. 6.4]). Therefore, $\tilde{\Theta}$ is a (global) diffeomorphism by Corollary 6.9 of [43].

Suppose now that $\bar{\omega} \neq 0$, set any $i: 1 \leq i \leq n$ and observe that for any choice of $\bar{\theta}_{1}, \ldots, \bar{\theta}_{i-1} \in[-\pi / 2, \bar{\pi} / 2]$, the map

$$
\alpha_{\bar{\theta}_{1}, \ldots, \bar{\theta}_{i-1}}:\left[-\frac{\pi}{2}, \frac{\pi}{2}\right] \rightarrow\left[-\frac{\pi}{2}, \frac{\pi}{2}\right]
$$

defined by $\alpha_{\bar{\theta}_{1}, \ldots, \bar{\theta}_{i-1}}\left(\theta_{i}\right)$ $\arg \left(\Pi_{i}\left(\tilde{\Theta}^{-1}\left(\bar{\theta}_{1}, \ldots, \bar{\theta}_{i-1}, \theta_{i}, 0, \ldots, 0\right)\right)-\left(\begin{array}{c}0 \\ 1 / \bar{\omega}\end{array}\right)\right)+\pi / 2$, is continuous, therefore it has a fixed point $\bar{\theta}_{i}$ that is

$\bar{\theta}_{i}=\arg \left(\Pi_{i}\left(\tilde{\Theta}^{-1}\left(\bar{\theta}_{1}, \ldots, \bar{\theta}_{i-1}, \bar{\theta}_{i}, 0, \ldots, 0\right)\right)-\left(\begin{array}{c}0 \\ \frac{1}{\bar{\omega}}\end{array}\right)\right)+\frac{\pi}{2}$.

Therefore, iterating on $i=1, \ldots, n$, we can find $\bar{\theta}=$ $\left(\bar{\theta}_{1}, \ldots, \bar{\theta}_{n}\right)^{T} \in[-\pi / 2, \pi / 2]^{n}$ such that (69) is verified for any $i=1, \ldots, n$. Note that $\forall\left(\theta_{1}, \ldots, \theta_{n}\right)^{T} \in[-\pi / 2, \pi / 2]^{n}$

$\partial_{\theta_{j}}\left(\Pi_{i}\left(\tilde{\Theta}^{-1}\left(\theta_{1}, \ldots, \theta_{n}\right)\right)\right)=0, \forall i=1, \ldots, n-1, \forall j=i, \ldots, n$

by (3) and the implicit function theorem. Therefore, $\Pi_{i}\left(\tilde{\Theta}^{-1}\left(\bar{\theta}_{1}, \ldots, \bar{\theta}_{i}, 0, \ldots, 0\right)\right)=\Pi_{i}\left(\tilde{\Theta}^{-1}\left(\bar{\theta}_{1}, \ldots, \bar{\theta}_{n}\right)\right)$, $\forall i=1, \ldots, n$ which implies by (69) that

$\bar{\theta}_{i}=\arg \left(\Pi_{i}\left(\tilde{\Theta}^{-1}\left(\bar{\theta}_{1}, \ldots, \bar{\theta}_{n}\right)\right)-\left(\begin{array}{c}0 \\ \frac{1}{\bar{\omega}}\end{array}\right)\right)+\frac{\pi}{2}, \forall i=1, \ldots, n$. Then $\bar{\xi}=\tilde{\Theta}^{-1}\left(\bar{\theta}_{1}, \ldots, \bar{\theta}_{n}\right)$ verifies (35) since $\bar{\theta}_{i}=\Theta_{i}(\bar{\xi})$, $\forall i=1, \ldots, n$. Hence, 2 ) holds.

\section{REFERENCES}

[1] R. Olfati-Saber, J. A. Fax, and R. M. Murray, "Consensus and cooperation in networked multi-agent systems," Proc. IEEE, vol. 95, no. 1, pp. 215-233, Jan. 2007.

[2] R. M. Murray, "Recent research in cooperative control of multivehicle systems," ASME J. Dyn. Syst. Meas. Control, vol. 129, no. 5, pp. 571-583, 2007.

[3] V. Kumar, D. Rus, and G. S. Sukhatme, "Networked Robots," in Handbook of Robotics, B. Siciliano and O. Khatib, Eds. New York: Springer, 2008, ch. 41, pp. 943-958.

[4] M. Mesbahi and M. Egerstedt, Graph Theoretic Methods in Multiagent Networks. Princeton, NJ: Princeton Univ. Press, 2010.

[5] F. Bullo, J. Cortés, and S. Martínez, Distributed Control of Robotic Networks. Princeton, NJ: Princeton Univ. Press, 2009.

[6] P. Ögren, E. Fiorelli, and N. E. Leonard, "Cooperative control of mobile sensor networks: Adaptive gradient climbing in a distributed environment," IEEE Trans. Autom. Control, vol. 49, no. 8, pp. 1292-1302, Aug. 2004.

[7] P. Tabuada, G. J. Pappas, and P. Lima, "Motion feasibility of multiagent formations," IEEE Trans. Robot., vol. 21, no. 3, pp. 387-391, Jun. 2005.

[8] Z. Lin, B. Francis, and M. Maggiore, "Necessary and sufficient graphical conditions for formation control of unicycles," IEEE Trans. Autom. Control, vol. 50, no. 1, pp. 121-127, 2005.

[9] W. Dong and J. A. Farrell, "Cooperative control of multiple nonholonomic mobile agents," IEEE Trans. Autom. Control, vol. 53, no. 6, pp. 1434-1448, Jun. 2008.

[10] N. Michael and V. Kumar, "Planning and control of ensembles of robots with non-holonomic constraints," Int. J. Robot. Res., vol. 28, no. 8, pp. 962-975, 2009.

[11] N. Moshtagh, N. Michael, A. Jadbabaie, and K. Daniilidis, "Visionbased, distributed control laws for motion coordination of nonholonomic robots," IEEE Trans. Robot., vol. 25, no. 4, pp. 851-860, Aug. 2009.

[12] P. Yang, R. A. Freeman, and K. M. Lynch, "Multi-agent coordination by decentralized estimation and control," IEEE Trans. Autom. Control, vol. 53, no. 11, pp. 2480-2496, Nov. 2008.

[13] A. K. Das, R. Fierro, V. Kumar, J. P. Ostrowsky, J. Spletzer, and C. Taylor, "A vision-based formation control framework," IEEE Trans. Robot. Autom., vol. 18, no. 5, pp. 813-825, May 2002. 
[14] T. Balch and R. C. Arkin, "Behavior-based formation control for multirobot teams," IEEE Trans. Robot. Autom., vol. 14, no. 6, pp. 926-939, Jun. 1998

[15] M. J. Matarić and F. Michaud, "Behavior-based systems," in Handbook of Robotics, B. Siciliano and O. Khatib, Eds. New York: Springer, 2008, ch. 38, pp. 891-906.

[16] K. H. Tan and M. A. Lewis, "High precision formation control of mobile robots using virtual structures," Auton. Robot., vol. 4, no. 4, pp. 387-403, 1997.

[17] M. Egerstedt and X. Hu, "Formation constrained multi-agent control," IEEE Trans. Robot. Autom., vol. 17, no. 6, pp. 947-951, Dec. 2001.

[18] S. Mastellone, D. M. Stipanovic, C. R. Graunke, K. A. Intlekofer, and M. W. Spong, "Formation control and collision avoidance for multiagent non-holonomic systems: Theory and experiments," Int. J. Robot. Res., vol. 27, no. 1, pp. 107-126, 2008

[19] H. G. Tanner, G. J. Pappas, and V. Kumar, "Leader-to-formation stability," IEEE Trans. Robot. Autom., vol. 20, no. 3, pp. 443-455, Jun. 2004.

[20] N. Léchevin, C. A. Rabbath, and P. Sicard, "Trajectory tracking of leader-follower formations characterized by constant line-of-sight angles," Automatica, vol. 42, no. 12, pp. 2131-2141, 2006.

[21] R. Vidal, O. Shakernia, and S. Sastry, "Following the flock: Distributed formation control with omnidirectional vision-based motion segmentation and visual servoing," IEEE Rob. Autom. Mag., vol. 11, no. 4, pp. 14-20, Dec. 2004.

[22] T. Eren, P. N. Belhumeur, B. D. O. Anderson, and A. S. Morse, "A framework for maintaining formations based on rigidity," in Proc. 15th IFAC World Congr., 2002, pp. 2752-2757.

[23] R. Olfati-Saber and R. M. Murray, "Graph rigidity and distributed formation stabilization of multi-vehicle systems," in Proc. 41 st IEEE Conf. Dec. Control, 2002, vol. 3, pp. 2965-2971.

[24] L. Asimow and B. Roth, "The rigidity of graphs II," J. Math. Anal. Appl., vol. 68, no. 1, pp. 171-190, 1979.

[25] J. Aspnes, T. Eren, D. Goldenberg, A. S. Morse, W. Whiteley, Y. R. Yang, B. D. O. Anderson, and P. Belhumeur, "A theory of network localization," IEEE Trans. Mobile Comput., vol. 5, no. 12, pp. 1663-1678, Dec. 2006.

[26] M. Cao, A. S. Morse, and B. D. O. Anderson, "Sensor network localization with imprecise distances," Syst. Control Lett., vol. 55, no. 11, pp. 887-893, 2006.

[27] C. Yu, J. M. Hendrickx, B. Fidan, B. D. O. Anderson, and V. D. Blondel, "Three and higher dimensional autonomous formations: Rigidity, persistence and structural persistence," Automatica, vol. 43, no. 3, pp. 387-402, 2007.

[28] B. D. O. Anderson, C. Yu, B. Fidan, and J. Hendrickx, "Rigid graph control architectures for autonomous formations," IEEE Control Syst. Mag., vol. 28, no. 6, pp. 48-63, Dec. 2008.

[29] L. Krick, M. E. Broucke, and B. A. Francis, "Stabilisation of infinitesimally rigid formations of multi-robot networks," Int. J. Control, vol. 82 , no. 3, pp. 423-439, 2009.

[30] F. Dörfler and B. A. Francis, "Geometric analysis of the formation problem for autonomous robots," IEEE Trans. Autom. Control, vol. 55, no. 10, pp. 2379-2384, Oct. 2010.

[31] J. Cortés, "Global and robust formation-shape stabilization of relative sensing networks," Automatica, vol. 45, no. 12, pp. 2754-2762, 2009.

[32] R. Sepulchre, D. A. Paley, and N. E. Leonard, "Stabilization of planar collective motion: All-to-all communication," IEEE Trans. Autom. Control, vol. 52, no. 5, pp. 811-824, May 2007.

[33] S. H. Strogatz, "From Kuramoto to Crawford: Exploring the onset of synchronization in populations of coupled oscillators," Physica D, vol. 143, no. 1-4, pp. 1-20, 2000.

[34] R. Sepulchre, D. A. Paley, and N. E. Leonard, "Stabilization of planar collective motion with limited communication," IEEE Trans. Autom. Control, vol. 53, no. 3, pp. 706-719, Mar. 2008.

[35] D. J. Klein and K. A. Morgansen, "Controlled collective motion for trajectory tracking," in Proc. Amer. Control Conf., 2006, pp. 5269-5275.

[36] D. J. Klein, E. Lalish, and K. A. Morgansen, "On controlled sinusoidal phase coupling," in Proc. Amer. Control Conf., 2009, pp. 616-622.

[37] C. Belta and V. Kumar, "Abstraction and control for groups of robots," IEEE Trans. Robot., vol. 20, no. 5, pp. 865-875, Oct. 2004.

[38] E. W. Justh and P. S. Krishnaprasad, "Equilibria and steering laws for planar formations," Syst. Control Lett., vol. 52, no. 1, pp. 25-38, 2004.

[39] L. Consolini, F. Morbidi, D. Prattichizzo, and M. Tosques, "Leaderfollower formation control of nonholonomic mobile robots with input constraints," Automatica, vol. 44, no. 5, pp. 1343-1349, 2008.
[40] L. Consolini, F. Morbidi, D. Prattichizzo, and M. Tosques, "Stabilization of a hierarchical formation of unicycle robots with velocity and curvature constraints," IEEE Trans. Robot., vol. 25, no. 5, pp. 1176-1184, Oct. 2009.

[41] P. B. S. Lissaman and C. A. Shollenberger, "Formation flight of birds," Science, vol. 168, no. 3934, pp. 1003-1005, 1970.

[42] J. M. Lee, Introduction to Smooth Manifolds, ser. Graduate texts in mathematics. New York: Springer, 2003.

[43] E. L. Lima, Fundamental Groups and Covering Spaces. Boca Raton, FL: A. K. Peters, 2003

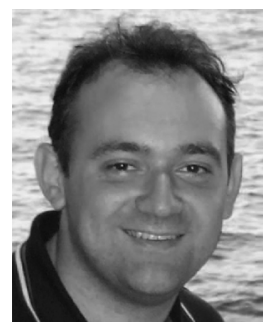

Luca Consolini (S'99-M'06) was born in 1976. He received the laurea degree in electronic engineering from the University of Parma, Parma, Italy, in 2000 and the Ph.D. degree from the University of Parma in 2005 under the supervision of prof. Aurelio Piazzi.

$\mathrm{He}$ is an Assistant Professor in the Dipartimento di Ingegneria dell'Informazione, University of Parma. In 2001 and 2002, he was a Visiting Scholar at the University of Toronto, Toronto, ON, Canada, collaborating with Prof. M. Maggiore. His main research topics are dynamic inversion for nonlinear systems, tracking and path following, formation control, and the study of virtual constraints for mechanical systems.

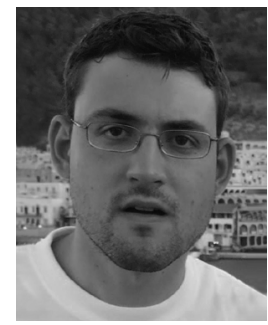

Fabio Morbidi (S'07-A'09) received the M.S. degree in computer engineering and the Ph.D. degree in robotics and automation from the University of Siena, Siena, Italy, in 2005 and 2009, respectively.

$\mathrm{He}$ was a Visiting Scholar at the Center for Control, Dynamical Systems and Computation, University of California, Santa Barbara, for six months in 2007-2008. He held postdoctoral positions at the University of Siena and at Northwestern University, Evanston, IL, from January 2009 to September 2010. Since October 2010, he has been a Postdoctoral Fellow in the Department of Computer Science and Engineering, University of Texas at Arlington. His research interests include multi-agent systems, formation control, robot vision, and geometric control.

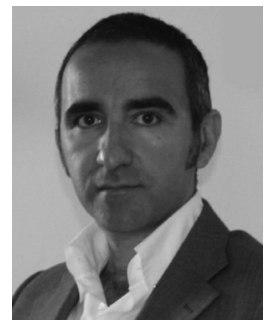

Domenico Prattichizzo (S'93-M'95) received the M.S. degree in electronics engineering and Ph.D. degree in robotics and automation from the University of Pisa, Pisa, Italy, in 1991 and 1995, respectively.

In 1994, he was a Visiting Scientist at the Artificial Intelligence Laboratory, Massachusetts Institute of Technology, Cambridge. Since 2002, he has been an Associate Professor of Robotics at the University of Siena, Siena, Italy. Since 2009, he has been a Scientific Consultant to Istituto Italiano di Tecnologia (IIT), Genova, Italy. He is coeditor of two books by STAR, Springer $(2003,2005)$. His research interests are in haptics, grasping and dexterous manipulation, computer vision, multi-agent systems, and geometric control theory. He is the author or coauthor of more than 160 papers in the area of robotics and automatic control.

Prof. Prattichizzo has been an Associate Editor-in-Chief of the IEEE TRANSACTIONS ON HAPTICS since 2007. From 2003 to 2007, he was an Associate Editor of the IEEE TRANSACTIONS ON RoBOTICS and IEEE TRANSACTIONS ON CONTROL Systems TeChNOLOGY. Since 2006, he has been vice-chair for special issues and workshops of the IEEE Technical Committee on Haptics. From 2006 to 2010, he was chair of the Italian Chapter of the IEEE RAS, awarded with the IEEE 2009 Chapter of the Year Award.

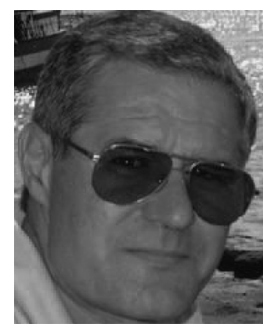

Mario Tosques was born in Pescara, Italy, in 1948. He graduated in mathematics from the University of Pisa, Pisa, Italy, in 1971 under the supervision of Prof. A. Andreotti.

He collaborated with professors E. De Giorgi and A. Marino. He is currently a Full Professor of mathematical analysis in the Department of Civil Engineering, University of Parma, Parma, Italy. His research interests lie in the areas of nonsmooth evolution equations and nonlinear control. 